\title{
Multi-Relay Selection in Energy-Harvesting Cooperative Wireless Networks: Game-Theoretic Modeling and Analysis
}

\author{
Mohammed W. Baidas - Emad Alsusa - Motasem Alfarra - Mubarak \\ Al-Mubarak
}

Received: date / Accepted: date

\begin{abstract}
This paper studies distributed multi-relay selection in energy-harvesting cooperative wireless networks and models it as an Indian Buffet Game (IBG). Particularly, the IBG is utilized to model the multirelay selection decisions of network source nodes, while account for negative network externality. Two scenarios are considered: (1) constrained selections (CS), and (2) unconstrained selections (US). In the former scenario, each source is constrained to a maximum number of relay selections; while in the latter scenario, the source nodes can select as many relays as possible. Since the relays are energy-harvesting - and thus intermittently harvest random amounts of energy - the accumulated energy at each relay is unknown to the source nodes, leading to uncertain relays' energy states. In turn, a non-Bayesian learning (NBL) algorithm is devised for the source nodes to learn the relays' energy states. After that, two distributed best-response (BR) recursive algorithms, namely BR-CS and BR-US, are proposed to allow the source nodes to make multi-relay selection decisions, while guaranteeing subgame perfect Nash equilib-
\end{abstract}

This work is partially supported by the Kuwait Foundation for the Advancement of Sciences (KFAS), under project code PN17-15EE-02.

Mohammed W. Baidas · Mubarak Al-Mubarak

Department of Electrical Engineering, College of Engineering and Petroleum, Kuwait University, Kuwait

E-mail:

m.baidas@ku.edu.kw,

mubarak.almubarak@grad.ku.edu.kw

Emad Alsusa

School of Electrical and Electronic Engineering, University of Manchester, Manchester, United Kingdom

E-mail: e.alsusa@manchester.ac.uk

Motasem Alfarra

Department of Electrical Engineering, King Abdullah University of Science and Technology, Thuwal, Saudi Arabia

E-mail: motasem.alfarra@kaust.edu.sa rium. Simulation results are presented to verify the efficacy of the proposed distributed NBL and multi-relay selection algorithms. Specifically, the NBL is shown to efficiently learn the true relays' energy states. More importantly, the BR-CS algorithm is shown to be comparable to the centralized multi-relay selection - and superior to other relay selection schemes - in terms of network sum-rate improvement (and utility). Lastly, the number of relay selections of the BR-CS algorithm must be constrained to the minimum so as to reduce complexity and fully exploit diversity gains.

Keywords Cooperation - Distributed · Energyharvesting · Game theory · Learning · Multi-relay selection

\section{Introduction}

Energy-harvesting (EH) has emerged as a promising alternative energy source to prolong the lifetime of energy-constrained wireless networks [1]. In particular, energy can be harvested from a variety of natural renewable sources within the surrounding environment such as solar, thermal and mechanical. With the recent developments in hardware design, energyharvesting networks are expected to be practically realizable and autonomous, with a potentially infinite lifetime [2]. However, to take full advantage of such networks, the intermittent and time-varying nature of energy arrivals at each network node must be cautiously managed. On the other hand, cooperative relay networks have been proposed to extend network coverage, exploit diversity gains and improve capacity [3]. However, due the finite battery-capacity at network source nodes, it is essential that relays with $\mathrm{EH}$ capabilities 
be optimally selected, while taking into account the stochastic nature of harvested energy.

Recently, several research works have considered the application of game-theoretic solutions for single- and multi-relay selection and cooperation in wireless networks. For instance, in [4], the author proposed a gametheoretic framework based on the iterated prisoner's dilemma (IPD) to model the repeated dynamic interactions of ad-hoc wireless networks with multiple source and amplify-and-forward (AF) relay nodes. Moreover, a reinforcement Q-learning algorithm is proposed to allow network nodes to adapt and optimally play the IPD game against other nodes with known and unknown strategies so as to achieved their maximum expected return values. The authors in [5] studied the problem of distributed multi-relay selection in one-to-many cooperative wireless via a political coalition formation game framework. In particular, a distributed algorithm has been proposed for the selection of a subset of decodeand-forward (DF) relays that is self-enforcing and stable to win against any other potential coalition while maximizing the network sum-rate. Moreover, a party formation solution was proposed to study the tradeoff between network sum-rate and stability. In [6], the authors studied the problem of joint cost-sharing and multi-relay selection in two-way AF relay networks with two source nodes via a repeated Bayesian Stackelberg game. Specifically, the two source nodes submit their payment offers to the relays that maximize the network power efficiency; whereas the cost of the relays participating in the game is covered by the promised payments. Moreover, Bayesian strategies along with a belief update system have been proposed to maximize the expected utility of players and achieve a perfect Bayesian equilibrium. Also, the authors proposed a stable game cost factor that offers tradeoff between network performance and complexity. In [7], a multi-relay selection scheme for energy-harvesting cooperative networks with a single source-destination pair is studied via a repeated Stackelberg game with only statistical knowledge of channel gains and energy profiles. Particularly, AF relays are selected based on repeated interactions with the source, which updates its payment offers to sequentially induce utility-maximizing relays to participate in the transmission. Moreover, the source applies Bayesian inference based on statistical knowledge of channel gains and energy profiles to select its strategy and update its beliefs, ultimately achieving perfect Bayesian equilibrium. The authors in [8] studied the problem of distributed single-relay selection via a Chinese restaurant game model, which incorporates negative network externality due to the potential sharing of an AF relay node among several source nodes. Two cases have been studied based on perfect and imperfect relay transmit power knowledge. In the first case, a distributed relay selection algorithm was proposed and shown to converge to a Nash equilibrium grouping; while in the second case, a reinforcement learning algorithm was proposed to allow network nodes to select expected rate-maximizing relays. In [9], distributed singlerelay selection is studied from a game-theoretic perspective. In particular, the authors study the case where a relay may be shared by multiple source-destination pairs, such that the total throughput is optimized. Also, the relay selection problem is modeled as a congestion game and a stochastic learning automata-based solution is proposed to obtain the NE.

Another line of work consider generic relay selection in energy-harvesting cooperative networks. For example, in [10], the authors consider several optimal power allocation and relay selection strategies - with and without energy cooperation - for signal-to-noise ratio (SNR) maximization. Specifically, optimal polynomial-time complexity solution procedures are proposed, where it has been shown that energy cooperation is effective in improving end-to-end SNR when augmented with the power allocation and relay selection strategies. The authors in [11] propose an energy-efficient scheme in a multirelay network with energy-harvesting relays. In particular, an optimal power allocation algorithm based on directional water-filling is proposed, and then an energyefficient relay selection and session grouping scheme is devised based on quantum particle swarm optimization. In [12], the authors analyze the outage probability of partial relay selection and optimal relay selection schemes in energy-harvesting networks over nonidentical Rayleigh fading channels. Specifically, closedform approximations of the outage probability under both schemes are derived and validated. The authors in [13] propose a DF relay selection protocol, where the relay with the highest harvested energy is selected. Moreover, an analytical expression for the outage probability and its lower-bound over Rayleigh fading channels are derived. In [14], several energy-harvesting DF relay selection schemes are considered. Specifically, first-hop SNR, second-hop SNR and end-to-end SNR maximizing relay selection schemes are proposed. Additionally, exact outage probability of the proposed schemes is given. Distributed multi-relay selection in ATF energyharvesting relay networks is studied in [15]. Specifically, the authors propose an energy threshold-based multirelay selection scheme, which can be implemented in a fully distributed manner to switch between energyharvesting and information-forwarding modes. Moreover, a heuristic approach to optimize the energy threshold for each relay is proposed. In [16], alternate $\mathrm{EH}$ 
and relaying for AF networks is considered. Particularly, the authors propose the use of two relays, where a relay harvests energy, while the other relay participates in cooperative data transmission. Outage probability and throughput analyses are derived, where it has been shown that the proposed scheme outperforms single-relay systems in terms of throughput. A joint power allocation, time-switching (TS) factor, and relay selection scheme is proposed in [17] for an EH twoway relay network. To be specific, a joint resource allocation algorithm to maximize the minimum instantaneous rate is devised. Additionally, the closed-form transmit power solution of the source nodes and the optimal TS factor at the relay are obtained. It has been shown that the proposed algorithm outperforms the traditional schemes as well as the power-splitting scheme. In [18], two relay selection methods are proposed to enhance the outage performance for EH twoway relaying protocols in multi-relay DF networks. In particular, the partial relay selection method selects a relay based on the partial channel state information (CSI), while the opportunistic relay selection method maximizes the end-to-end SNR. Furthermore, exact and asymptotic expressions for the system outage probability over Rayleigh fading channels are derived. It has been demonstrated that the proposed methods outperform random relay selection as well as the single-relay protocol. Incremental relaying with energy-harvesting relay selection is considered in [19]. In particular, the cases of no combining, selection combining, and maximal ratio combining are considered. In turn, exact and asymptotic expressions are derived, and a linear search algorithm to obtain the optimal time-switching ratio is proposed. It has been shown that the proposed scheme is superior to that of direct transmission for a given average transmit power. In [20], the authors consider the performance EH relays with the best relay selection (BRS). Specifically, it has been shown that spectral efficiency degradation occurs due to the EH relaying when compared with conventional relaying. On the other hand, EH relaying has been shown to provide other gains, such as increasing network lifetime, improving energy-efficiency, and decreasing energy consumption and operational cost.

In practice, the assumption that all the temporallyvarying harvested energy at each relay node to be known network-wide is not feasible due to the excessive communication and signalling overheads. Additionally, although increasing the number of selected relays improves the diversity gains, significant computational and communication delays are incurred. Therefore, constraining the number of relays that can be selected may provide a reasonable tradeoff between complexity and per- formance. Moreover, for a source node to select relays, it must observe the prior and current relay selection decisions of the other source nodes, determine the expected utility/achievable rate of each potential relay, and then select the relay (or set of relays) that maximizes its expected utility/rate.

In this paper, the problem of multi-relay selection in energy-harvesting cooperative wireless networks is modeled as a sequential Indian Buffet Game (IBG) [21], which originated from the Indian Buffet process (IBP) [22]. The IBP assumes that there exists an infinite number of dishes, and each customer can order a number of dishes following Poisson process/distribution. However, the IBP does not take into account the customers' strategic behavior or utility-maximizing decision making. Hence, by incorporating utility functions and customers' strategic aim to maximize their utilities, the IBP is transformed into the IBG. Particularly, the IBG is suitable for modeling scenarios, where customers would like to select multiple dishes, while incorporating social learning, strategic decision making, and negative network externality. Additionally, the IBG allows sequential decision making, which is specifically important in the case where previous customer decisions are observed, while the following customers' decisions can be predicted. More importantly, and unlike the IBP, the IBG can be adapted to two cases: (1) with budget constraint, and (2) without budget constraint. Moreover, the IBG incorporates social learning, such that optimal decisions can be made so as to maximize expected customers' utilities. By analogy, in this work, the aim is to allow source nodes to make strategic expected utilitymaximizing relay selection decisions. Specifically, each rational source node sequentially selects utility-maximizing relays from a set of available energy-harvesting AF relays, while taking into account negative network externality (i.e. the potential sharing of a relay by multiple source nodes). To account for the uncertainty of the harvested energy at each relay, a non-Bayesian learning (NBL) algorithm is proposed, which is shown to have a polynomial-time complexity, and thus can be executed efficiently. Moreover, two relay selection scenarios are considered: (1) constrained selections (CS), and (2) unconstrained selections (US). In the former scenario, each source node is constrained to a maximum number of relay selections; while in the latter scenario, the source nodes can select as many relays as possible. After that, two distributed recursive bestresponse (BR) algorithms are proposed, namely BRCS and BR-US, which guarantee subgame perfect Nash equilibrium. In particular, the proposed algorithms allow network source nodes to make multiple relay selections under unknown relays' energy states using the 
NBL algorithm, such that their relay selection decisions are optimized in terms of their expected utilities, while incorporating negative network externality. It is noteworthy that the IBP/IBG can be applied to a variety of network applications and scenarios. For instance, in [23], the IBP has been applied for device-to-device communications, with the aim of modeling the distribution of users' contents in online social networks, such that packet transmission is improved while offloading network traffic. Moreover, the Indian Buffet learning has been applied to model the social influence in online social networks, where users are allowed to select multiple contents. Also, the IBG has been utilized for learning content popularity distribution and predicting users' selections. In turn, a traffic offloading algorithm has been proposed to obtain the distribution of contents requests and probabilities of each content to be requested, ultimately improving the network data rate.

To the best of our knowledge, no prior work has modeled and analyzed distributed multi-relay selection in energy-harvesting cooperative wireless networks with constrained and unconstrained relay selections. Hence, the main contributions of this work are summarized as:

- Modeled the multi-relay selection problem of energyharvesting AF relays as an Indian Buffet Game.

- Proposed a non-Bayesian learning algorithm so as to learn the true relays' energy states.

- Proposed two recursive best-response distributed algorithms that can select utility-maximizing relays, while accounting for negative network externality.

- Demonstrated that the BR-CS algorithm outperforms (in terms of network sum-rate improvement and utility) its BR-US counterpart algorithm when the number of relay selections is constrained to less than the total number of available relays; however, at the expense of higher complexity.

- Compared the proposed BR-CS and BR-US algorithms to learning-based, myopic and random multirelay selection schemes, where it is shown that the BR-CS algorithm is superior to the aforementioned schemes, and yields comparable network sum-rate improvement (and utility) to the centralized multirelay selection.

- Demonstrated that the number of relay selections of the BR-CS algorithm must be constrained to the minimum so as to reduce complexity and fully exploit diversity gains.

It is noteworthy that the proposed algorithms can be applied to green cooperative wireless networks with renewable energy-powered relays (i.e. with no grid infrastructure available) [24,25]. Particularly, such algorithms can be used in cellular networks to improve quality-of- service, and extend network coverage, while minimizing energy and computational costs [26]. Moreover, the proposed algorithms can be used to achieve cognitive relaying so as to balance energy-efficiency, data transmission, network capacity [27]. Generally speaking, the proposed game-theoretic framework with non-Bayesian learning can be applied across different network settings with renewable-energy-powered relay stations.

In the remainder of this paper, Section 2 presents the system model; while Section 3 models the multirelay selection problem as an Indian buffet game. In Section 4, the relay selection game with constrained selections is presented, while Section 5 presents the unconstrained selections game. Section 7 presents the simulation results of the proposed relay selection algorithms, while Section 8 draws the conclusions.

\section{System Model}

Let us consider an ad-hoc wireless network with $N$ source nodes and $K$ energy-harvesting single-antenna AF relay nodes and a single destination node $D$. Each source node $S_{i}, i \in\{1,2, \ldots, N\}$, communicates its data symbol $x_{i}$ to the destination via one or more relay nodes $R_{k}$, for $k \in\{1,2, \ldots, K\}$. The channel between any two nodes is modeled as a quasi-static narrowband Rayleigh fading channel with zero-mean $N_{0}$-variance additive white Gaussian noise (AWGN). In particular, $h_{i, k} \sim \mathcal{C N}\left(0, \sigma_{i, k}^{2}\right), h_{k, d} \sim \mathcal{C N}\left(0, \sigma_{k, d}^{2}\right)$, and $h_{i, d} \sim$ $\mathcal{C N}\left(0, \sigma_{i, d}^{2}\right)$ are defined as the channel coefficients of the source-relay, relay-destination, and source-destination links, respectively ${ }^{1}$. Moreover, $\sigma_{i, k}^{2}=d_{i, k}^{-\nu}, \sigma_{k, d}^{2}=d_{k, d}^{-\nu}$, $\sigma_{i, d}^{2}=d_{i, d}^{-\nu}$ are the channel gains of the respective links, with $d_{i, k}, d_{k, d}$, and $d_{i, d}$ being the inter-node distances and $\nu$ is the path-loss exponent. On the other hand, each source node $S_{i}$ is assigned a signature waveform $c_{i}(t)$ to allow for multiuser detection at the destination. Specifically, the cross-correlation coefficient between any two waveforms $c_{i}(t)$ and $c_{j}(t)$ (for $i \neq j$ ) is denoted $0 \leq \rho_{i, j} \leq 1$, where $\rho_{i, i}=1, \forall i \in\{1,2, \ldots, N\}$. Without loss of generality, it is assumed that $\rho_{i, j}=\rho$, $\forall i, j \in\{1,2, \ldots, N\}$ and $i \neq j$.

In this work, the data transmission and energy harvesting models proposed in [10] are adopted. Specifically, data transmission between the source nodes and the destination is achieved in a time-division multipleaccess (TDMA) fashion with a total of $N+1$ equallength unit-duration time-slots ${ }^{2}$ split over two phases:

\footnotetext{
1 Perfect CSI is assumed at the source and relay nodes.

2 Power and energy terms can be used interchangeably due to the normalized time-slots.
} 
$\mathbb{P}\left[\xi_{r_{k}}^{\zeta}=\xi \mid M\right]= \begin{cases}\frac{1}{\mathcal{E}_{r_{k}}^{\max }} \sum_{j=1}^{\mathcal{E}_{r_{k}}^{\max }} \mathbb{P}\left[\xi_{r_{k}}^{\zeta}=\xi-j \mid M-1\right], & \xi \in\left\{M, M+1, \ldots, M \mathcal{E}_{r_{k}}^{\max }\right\}, \\ 0, & \text { otherwise }\end{cases}$

(i) broadcasting phase ( $N$ time-slots), and (ii) cooperation phase (1 multiple-access time-slot). Moreover, let $\zeta \geq 1$ represent a communication frame that consists of $N+1$ time-slots. In each frame, the relays harvest energy from the $N$ previous time-slots (i.e. the broadcasting phase), and subsequently utilize it for the cooperative transmission (in the cooperation phase) ${ }^{3}$. Furthermore, channels experience block-fading and thus remain constant during a communication frame, but change from one frame to another.

\subsection{Energy Model}

Energy arrivals at the relays are modeled as Poisson processes with rates $\lambda_{r_{k}}$ per time-slot, for $k \in\{1,2, \ldots, K\}$ Specifically, let $\mathcal{N}_{r_{k}}^{\zeta}$ represent the number of energy arrivals up to and including the $N^{t h}$ time-slot of frame $\zeta$, $\forall \zeta \geq 1$. Additionally, let $\tau_{r_{k}, m}^{\zeta}$ (for $m=1,2, \ldots$ ) be the random time-instants of the energy arrivals at relay $R_{k}$ in frame $\zeta$, while the energy arrival values at the timeinstants $\tau_{r_{k}, m}^{\zeta}$ can be modeled as independent and identically distributed (i.i.d.) discrete uniformly distributed random variables ${ }^{4}$, such that $\mathcal{E}_{r_{k}, m}^{\zeta} \sim U\left[1, \mathcal{E}_{r_{k}}^{\max }\right]$, where $\mathcal{E}_{r_{k}}^{\max }$ is the maximum energy arrival value at that relay, and $\mathbb{P}\left[\mathcal{E}_{r_{k}, m}^{\zeta}=\xi\right]=\frac{1}{\mathcal{E}_{r_{k}}^{\max }}, \forall \xi \in\left\{1,2, \ldots, \mathcal{E}_{r_{k}}^{\max }\right\}$. Hence, the total harvested energy up to the $N^{\text {th }}$ timeslot in frame $\zeta$ at relay $R_{k}$ is given by

$\xi_{r_{k}}^{\zeta}=\sum_{m=1}^{\mathcal{N}_{r_{k}}^{\zeta}} \mathcal{E}_{r_{k}, m}^{\zeta}$

Also, it is assumed that each relay $R_{k}$ has a batterycapacity constraint $B_{\max }$, such that $\xi_{r_{k}}^{\zeta} \leq B_{\max }, \forall k \in$ $\{1,2, \ldots, K\}$.

Remark 1: For the Poisson process $\mathcal{N}_{r_{k}}^{\zeta}$ with rate $\lambda_{r_{k}}$, and for any $\zeta \geq 1$, the probability mass function (PMF) of the number of energy arrivals $\mathcal{N}_{r_{k}}^{\zeta}$ (over $N$ time-slots) is given by [30]

$\mathbb{P}\left[\mathcal{N}_{r_{k}}^{\zeta}=m\right]=\frac{\left(\lambda_{r_{k}} N\right)^{m} e^{-\lambda_{r_{k}} N}}{m !}, \quad$ for $m=0,1, \ldots$,

where it should be noted that $\mathbb{P}\left[\mathcal{N}_{r_{k}}^{\zeta}=0\right]>0$, and the expected value of $\mathcal{N}_{r_{k}}^{\zeta}$ is $\lambda_{r_{k}} N$.

\footnotetext{
3 Due to hardware limitations, it is assumed that no energy is harvested during the multiple-access time-slot, as the relays may be involved in cooperative transmission.

4 The case for i.i.d continuous uniform random variables can be straightforwardly incorporated $[28,29]$.
}

Remark 2: For the sum of $M \geq 2$ i.i.d discrete uniform random variables in frame $\zeta, \xi_{r_{k}}^{\zeta} \mid M=\mathcal{E}_{r_{k}, 1}^{\zeta}+$ $\mathcal{E}_{r_{k}, 2}^{\zeta}+\cdots+\mathcal{E}_{r_{k}, M}^{\zeta}\left(\right.$ with $\mathcal{E}_{r_{k}, m}^{\zeta} \sim U\left[1, \mathcal{E}_{r_{k}}^{\max }\right], \forall m \in$ $\{1,2, \ldots, M\})$, the conditional PMF can be expressed in recursive form as given in (3) [31, Eqs. (2.6-2.7)], where it should be noted that

$\mathbb{P}\left[\xi_{r_{k}}^{\zeta}=\xi \mid 1\right]= \begin{cases}\frac{1}{\mathcal{E}_{r_{k}}^{\max }}, & \xi \in\left\{1,2, \ldots, \mathcal{E}_{r_{k}}^{\max }\right\}, \\ 0, & \text { otherwise. }\end{cases}$

Moreover, by independence, the expected value of $\xi_{r_{k}}^{\zeta}$ conditional on the number of energy arrivals $M$ can be shown to be $M\left(\mathcal{E}_{r_{k}}^{\max }+1\right) / 2$.

Remark 3: The total harvested energy $\xi_{r_{k}}^{\zeta}$ at relay $R_{k}$ in frame $\zeta \geq 1$ is a compound Poisson process $[30,32]$, since $\mathcal{N}_{r_{k}}^{\zeta}$ is a Poisson process with rate $\lambda_{r_{k}}$ per time-slot over $N$ time-slots, and the energy arrivals $\mathcal{E}_{r_{k}, m}^{\zeta}(\forall m \geq 1)$ are i.i.d. discrete uniformly distributed random variables that are independent of $\mathcal{N}_{r_{k}}^{\zeta}$. Hence, the PMF of $\xi_{r_{k}}^{\zeta}$ is obtained as [32]

$p_{\xi_{r_{k}}}(\xi) \triangleq \mathbb{P}\left[\xi_{r_{k}}^{\zeta}=\xi\right]=\sum_{m=0}^{\infty} \mathbb{P}\left[\xi_{r_{k}}^{\zeta}=\xi \mid m\right] \cdot \mathbb{P}\left[\mathcal{N}_{r_{k}}^{\zeta}=m\right]$,

$\forall \xi=0,1, \ldots$, where $\mathbb{P}\left[\xi_{r_{k}}^{\zeta}=0 \mid 0\right]=1$. Lastly, it can be verified that $\sum_{\xi=0}^{\infty} p_{\xi_{r_{k}}^{\zeta}}(\xi) \rightarrow 1$, and the expected value of $\xi_{r_{k}}^{\zeta}$ can be obtained as $\lambda_{r_{k}} N\left(\mathcal{E}_{r_{k}}^{\max }+1\right) / 2$ [30].

\subsection{Data Transmission}

\subsubsection{Broadcasting Phase}

In the broadcasting phase, each source node $S_{i}$ broadcasts its data symbol $x_{i}^{\zeta}$ in its assigned time-slot, where the signals received at relay $R_{k}$ and the destination are expressed as [33]

$y_{i, k}^{\zeta}(t)=\sqrt{E_{B_{i}}^{\zeta}} h_{i, k}^{\zeta} x_{i}^{\zeta} c_{i}(t)+\eta_{i, k}^{\zeta}(t)$,

and

$y_{i, d}^{\zeta}(t)=\sqrt{E_{B_{i}}^{\zeta}} h_{i, d}^{\zeta} x_{i}^{\zeta} c_{i}(t)+\eta_{i, d}^{\zeta}(t)$,

respectively, where $E_{B_{i}}^{\zeta}$ is the broadcast transmit energy per symbol. Also, $\eta_{i, k}^{\zeta}(t)$ and $\eta_{i, d}^{\zeta}(t)$ are the AWGN processes at relay $R_{k}$ and the destination, respectively. The received signal-to-noise ratio (SNR) at the destination is expressed as

$\gamma_{i, d}=\frac{E_{B_{i}}^{\zeta}\left|h_{i, d}^{\zeta}\right|^{2}}{N_{0}}$ 
$\mathcal{R}_{i}^{\zeta}\left(\mathbf{E}_{C_{i}}^{\zeta}, \mathcal{I}_{i}^{\zeta}\right)=\frac{1}{N+1} \log _{2}\left(1+\frac{E_{B_{i}}^{\zeta}\left|h_{i, d}^{\zeta}\right|^{2}}{N_{0}}+\sum_{k=1}^{K} \mathcal{I}_{i, k}^{\zeta} \frac{E_{B_{i}}^{\zeta} E_{C_{i, k}}^{\zeta}\left|h_{i, k}^{\zeta}\right|^{2}\left|h_{k, d}^{\zeta}\right|^{2}}{N_{0} \varrho_{N}\left(E_{B_{i}}^{\zeta}\left|h_{i, k}^{\zeta}\right|^{2}+E_{C_{i, k}}^{\zeta}\left|h_{k, d}^{\zeta}\right|^{2}+N_{0}\right)}\right)$

$\mathcal{R}_{i}\left(\mathbf{E}_{R}^{\zeta}, \mathbf{n}_{R}^{\zeta}\right)=\frac{1}{N+1} \log _{2}\left(1+\frac{E_{B_{i}}^{\zeta}\left|h_{i, d}^{\zeta}\right|^{2}}{N_{0}}+\sum_{k=1}^{K} \mathcal{I}_{i, k}^{\zeta} \frac{E_{B_{i}}^{\zeta}\left(\frac{E_{r_{k}}^{\zeta}}{n_{r_{k}}^{\zeta}}\right)\left|h_{i, k}^{\zeta}\right|^{2}\left|h_{k, d}^{\zeta}\right|^{2}}{N_{0} \varrho_{N}\left(E_{B_{i}}^{\zeta}\left|h_{i, k}^{\zeta}\right|^{2}+\left(\frac{E_{r_{k}}^{\zeta}}{n_{r_{k}}^{\zeta}}\right)\left|h_{k, d}^{\zeta}\right|^{2}+N_{0}\right)}\right)$

At the end of this phase, each relay $R_{k}$ will have received $N$ signals $y_{i, k}^{\zeta}(t), \forall i \in\{1,2, \ldots, N\}$ and $\forall k \in$ $\{1,2, \ldots, K\}$.

\subsubsection{Cooperation Phase}

In this phase, each relay forms a linearly-coded signal as given by

$\mathcal{X}_{r_{k}}^{\zeta}(t)=\sum_{j=1}^{N} \mathcal{I}_{j, k}^{\zeta} \sqrt{E_{C_{j, k}}^{\zeta}} \beta_{j, k}^{\zeta} y_{j, k}^{\zeta} c_{j}(t)$

where $E_{C_{j, k}}^{\zeta}$ is the transmit energy assigned by $R_{k}$ to forward $S_{j}$ 's data; while $\beta_{j, k}^{\zeta}=\sqrt{\frac{1}{E_{B_{j}}^{\zeta}\left|h_{j, k}^{\zeta}\right|^{2}+N_{0}}}$ is a normalization factor. Moreover, $\mathcal{I}_{j, k}^{\zeta}$ is binary indicator function defined as

$\mathcal{I}_{j, k}^{\zeta}= \begin{cases}1, & \text { if source } S_{j} \text { selects relay } R_{k}, \\ 0, & \text { otherwise }\end{cases}$

Assuming perfect timing synchronization, all relays simultaneously transmit their signals $\mathcal{X}_{r_{k}}^{\zeta}(t)$, which are received at the destination as given by [33]

$y_{d}^{\zeta}(t)=\sum_{k=1}^{K} h_{k, d}^{\zeta} \mathcal{X}_{k}^{\zeta}(t)+\eta_{d}^{\zeta}(t)$,

where $\eta_{d}^{\zeta}(t)$ is the AWGN process at the destination. By applying multiuser detection and maximum ratio combining, the end-to-end SNR of source node $S_{i}$ can be shown to be [8]

$$
\begin{aligned}
\gamma_{i}^{\zeta}=\frac{E_{B_{i}}^{\zeta}\left|h_{i, d}^{\zeta}\right|^{2}}{N_{0}}+ & \\
& \sum_{k=1}^{K} \mathcal{I}_{i, k}^{\zeta} \frac{E_{B_{i}}^{\zeta} E_{C_{i, k}}^{\zeta}\left|h_{i, k}^{\zeta}\right|^{2}\left|h_{k, d}^{\zeta}\right|^{2}}{N_{0} \varrho_{N}\left(E_{B_{i}}^{\zeta}\left|h_{i, k}^{\zeta}\right|^{2}+E_{C_{i, k}}^{\zeta}\left|h_{k, d}^{\zeta}\right|^{2}+N_{0}\right)},
\end{aligned}
$$

where $\varrho_{N}$ is a noise amplification coefficient due to the use of signature waveforms, as given by

$\varrho_{N}=\frac{1+(N-2) \rho}{1+(N-2) \rho-(N-1) \rho^{2}}$.
In turn, the achievable rate of source node $S_{i}$ in communication frame $\zeta$ is obtained as given in (14), where

$\mathbf{E}_{C_{i}}^{\zeta}=\left[E_{C_{i, 1}}^{\zeta}, E_{C_{i, 2}}^{\zeta}, \ldots, E_{C_{i, K}}^{\zeta}\right]$,

and

$\mathcal{I}_{i}^{\zeta}=\left[\mathcal{I}_{i, 1}^{\zeta}, \mathcal{I}_{i, 2}^{\zeta}, \ldots, \mathcal{I}_{i, K}^{\zeta}\right]$

Remark 4: It is assumed that there is a maximum transmit energy constraint $E_{\text {max }}$ per node in each communication frame $\zeta$, such that $E_{B_{i}}^{\zeta} \leq E_{\max }, \forall i \in$ $\{1,2, \ldots, N\}$.

Remark 5: Due to the total energy constraint $E_{\max }$ per node, the total transmit power per relay $R_{k}$ in the cooperation phase is such that $\sum_{i=1}^{N} \mathcal{I}_{i, k}^{\zeta} E_{C_{i, k}}^{\zeta} \leq$ $\min \left(\xi_{r_{k}}^{\zeta}, E_{\max }\right) \triangleq E_{r_{k}}^{\zeta}, \forall k \in\{1,2, \ldots, K\}$.

According to Remark 5, if $\xi_{r_{k}}^{\zeta} \geq E_{\max }$, then $E_{r_{k}}^{\zeta}=$ $E_{\max }$ and any leftover harvested energy (i.e. $\Delta \xi_{r_{k}}^{\zeta}=$ $\left.\xi_{r_{k}}^{\zeta}-E_{\max }\right)$ will be stored in the battery for future use; otherwise, all the available harvested energy is utilized for cooperative transmission and no energy is leftover (i.e. $\Delta \xi_{r_{k}}^{\zeta}=0$ ). Therefore, in any communication frame $\zeta$, the total harvested energy must satisfy $\xi_{r_{k}}^{\zeta}=\min \left(\Delta \xi_{r_{k}}^{\zeta-1}+\xi_{r_{k}}^{\zeta}, B_{\max }\right), \forall k \in\{1,2, \ldots, K\}$.

Remark 6: Equal power allocation is assumed at each relay $R_{k}$, i.e. $E_{C_{i, k}}^{\zeta}=\frac{E_{r_{k}}^{\zeta}}{\sum_{i=1}^{N} \mathcal{I}_{i, k}^{\zeta}}, \forall i \in\{1,2, \ldots, N\}$. Thus, the greater the number of source nodes selecting a relay is, the lesser the allocated transmit energy per source node (i.e. negative network externality).

Now, let $n_{r_{k}}^{\zeta} \triangleq \sum_{i=1}^{N} \mathcal{I}_{i, k}^{\zeta}$ be the number of sources nodes requesting/selecting relay $R_{k}$, then $E_{C_{i, k}}^{\zeta}=\frac{E_{r_{k}}^{\zeta}}{n_{r_{k}}^{\zeta}}$, $\forall k \in\{1,2, \ldots, K\}$. Based on Remark 6, the achievable rate function in (14) is re-defined as given in (17), where $\mathbf{E}_{R}^{\zeta}=\left[E_{r_{1}}^{\zeta}, E_{r_{2}}^{\zeta}, \ldots, E_{r_{K}}^{\zeta}\right]$, and $\mathbf{n}_{R}^{\zeta}=\left[n_{r_{1}}^{\zeta}, n_{r_{2}}^{\zeta}, \ldots, n_{r_{K}}^{\zeta}\right]$. 


\section{Multi-Relay Selection as an Indian Buffet Game}

\subsection{Utility and Payment Functions}

The IBG model assumes that $N$ customers sequentially request dishes out of the available $K$ dishes, where each dish can be shared by multiple customers, and each customer can select multiple dishes. Moreover, the quality of each dish can be defined in terms of its size or deliciousness. In an analogous manner, the $N$ customers represent the $N$ source nodes, while the $K$ dishes are represented by the $K$ relays. Also, the quality of a dish is interpreted as the amount of available harvested energy $\xi_{r_{k}}^{\zeta}$ at relay $R_{k}$ in each communication frame $\zeta$. Therefore, the strategy set of each source node is given by a combination of requested/selected relays, as $\mathcal{S}=$ $\left\{\emptyset,\left\{R_{1}\right\},\left\{R_{2}\right\}, \ldots,\left\{R_{1}, R_{2}\right\}, \ldots,\left\{R_{1}, R_{2}, \ldots, R_{K}\right\}\right\}$ (i.e. $2^{K}$ possible choices). Since all source nodes are assumed to be rational and selfish - and thus aim at maximizing their own utilities by carefully selecting relays - the utility function of source $S_{i}$ when it selects relay $R_{k}$ can be defined as [34]

$\mathcal{U}_{i, k}\left(E_{r_{k}}^{\zeta}, n_{r_{k}}^{\zeta}\right)=\Delta \mathcal{R}_{i, k}\left(E_{r_{k}}^{\zeta}, n_{r_{k}}^{\zeta}\right)-\mathcal{P}_{i, k}\left(E_{r_{k}}^{\zeta}, n_{r_{k}}^{\zeta}\right)$,

where $\Delta \mathcal{R}_{i, k}\left(E_{r_{k}}^{\zeta}, n_{r_{k}}^{\zeta}\right)$ is the rate improvement due to the cooperation of relay $R_{k}$, as given by [34]

$\Delta \mathcal{R}_{i, k}\left(E_{r_{k}}^{\zeta}, n_{r_{k}}^{\zeta}\right)=\frac{1}{N+1} \log _{2}\left(1+\frac{\left(\frac{E_{r_{k}}^{\zeta}}{n_{r_{k}}^{\zeta}}\right) \cdot \Omega_{i, k}^{\zeta}}{\left(\frac{E_{r_{k}}^{\zeta}}{n_{r_{k}}^{\zeta}}\right)+\Upsilon_{i, k}^{\zeta}}\right)$,

where

$\Omega_{i, k}^{\zeta}=\frac{E_{B_{i}}^{\zeta}\left|h_{i, k}^{\zeta}\right|^{2}}{\varrho_{N}\left(E_{B_{i}}^{\zeta}\left|h_{i, d}^{\zeta}\right|^{2}+N_{0}\right)}$,

and

$\Upsilon_{i, k}^{\zeta}=\frac{E_{B_{i}}^{\zeta}\left|h_{i, k}^{\zeta}\right|^{2}+N_{0}}{\left|h_{k, d}^{\zeta}\right|^{2}}$,

respectively. On the other hand, $\mathcal{P}_{i, k}\left(E_{r_{k}}^{\zeta}, n_{r_{k}}^{\zeta}\right)$ is the payment source $S_{i}$ makes to relay $R_{k}$ in return for the cooperative service and utilization of harvested energy. In this work, the payment each source $S_{i}$ makes is expressed as

$\mathcal{P}_{i, k}\left(E_{r_{k}}^{\zeta}, n_{r_{k}}^{\zeta}\right)=\chi_{r_{k}} \cdot\left(\frac{E_{r_{k}}^{\zeta}}{n_{r_{k}}^{\zeta}}\right)$,

where $\chi_{r_{k}}$ is the price per unit energy of relay $R_{k}$.

Remark 7: It can straightforwardly be verified that the rate improvement and payment functions per source node $S_{i}$ are increasing in $E_{r_{k}}^{\zeta}$, but decreasing in $n_{r_{k}}$, $\forall i \in\{1,2, \ldots, N\}$ and $\forall k \in\{1,2, \ldots, K\}$.

Remark 8: If a source node does not select a relay, then its rate improvement will be zero, and thus its utility will also be zero. In this case, the source node achievable rate will be equivalent to that of direct transmission. Hence, each rational source node has an incentive to select at least one relay so as to improve its achievable rate via cooperative transmission, provided that the selected relay does not yield negative utility.

\subsection{Network Operation as a Repeated Game}

In such a dynamic network with stochastic energy arrivals, the source nodes may only have limited information about the quality/state of each relay node. For instance, some relay nodes may be close to the energy source, and thus can harvest energy at a higher rate with higher energy arrival values. On the other hand, other relays may be farther from the energy source, and thus may harvest energy at a lower rate with relatively lower energy arrival values. Moreover, since the energy arrivals at each relay are modeled as Poisson processes (as per subsection 2.1), then the total amount of harvested energy change from one communication frame to another. Therefore, it is essential for the source nodes to learn the true relays' energy states. In other words, the source nodes must construct a probabilistic belief regarding the relays' states through learning. To that end, let the relay states be defined as $\boldsymbol{\theta}=\left\{\theta_{r_{1}}, \theta_{r_{2}}, \ldots, \theta_{r_{K}}\right\}$, where $\theta_{r_{k}} \in \Theta$ is the state of relay $R_{k}$, and $\Theta$ is the finite set of all possible states. Moreover, $\theta_{r_{k}}$ represents the quality of the relay, which is assumed to remain the same throughout the network operation. In this work, a binary relay state is assumed for all $K$ relay nodes, such that $\theta_{r_{k}} \in\{G, B\} \triangleq \Theta, \forall k \in\{1,2, \ldots, K\}^{5}$. Particularly, when relay $R_{k}$ is in the Good $(G)$ state, it is characterized by energy arrivals $\lambda_{r_{k}, G}$ that are more frequent with higher energy values $\mathcal{E}_{r_{k}, G}^{\max }$; while the $B a d(B)$ state indicates less frequent energy arrivals $\lambda_{r_{k}, B}$ with lower values $\mathcal{E}_{r_{k}, B}^{\max }$ (i.e. $\lambda_{r_{k}, G}>\lambda_{r_{k}, B}$ and $\left.\mathcal{E}_{r_{k}, G}^{\max }>\mathcal{E}_{r_{k}, B}^{\max }\right)$. In other words, during communication frame $\zeta$, the distribution of the harvested energy $\xi_{r_{k}}^{\zeta}$ of relay $R_{k}$ follows $p_{\mathcal{E}_{r_{k}}^{\zeta}}\left(\xi \mid \theta_{r_{k}}\right)$, which is conditioned on state $\theta_{r_{k}}$ (as per Remark 3). However, the amount of harvested energy at each relay varies from one communication frame to another.

Since the relays' states $\boldsymbol{\theta} \in \Theta^{K}$ are unknown to all source nodes (i.e. they do not know if a relay is good or bad before selecting it), then in each communication frame, the source nodes make sequential decisions

5 In general, there could be more than two relay states, but this setting is beyond the scope of this paper. 
on which relays to select. Particularly, two main issues are to be considered: (1) the design of a learning algorithm to allow the source nodes to learn from each other's requests/selections, and (2) the best-response strategy of each source node given the estimated knowledge of relays' states. Hence, in each communication frame $\zeta$, the source nodes must construct a belief about each relay $R_{k}, \mathbf{p}_{r_{k}}^{\zeta}=\left\{p_{r_{k}}^{\zeta}\left(\theta_{r_{k}}\right), \theta_{r_{k}} \in \Theta\right\}$, where the beliefs about all $K$ relays' states can be expressed as $\mathbf{p}_{R}^{\zeta}=\left\{\mathbf{p}_{r_{1}}^{\zeta}, \mathbf{p}_{r_{2}}^{\zeta}, \ldots, \mathbf{p}_{r_{K}}^{\zeta}\right\}$. Moreover, all source nodes start with an initial "prior" belief $p_{r_{k}}^{(0)}\left(\theta_{r_{k}}\right)>0$ about the state of relay $R_{k}, \forall \theta_{r_{k}} \in \Theta$.

Each communication frame $\zeta$ takes the form of a repeated game, and is divided into three stages:

Stage 1: Sequential Best-Response Decision Making

In this stage, each source node makes relay selection decisions in a sequential manner, which are observed by all the other source nodes. Specifically, each source node $S_{i}$ 's decision is such that its expected utility is maximized at the current communication frame $\zeta$, which is based on the current belief $\mathbf{p}_{R}^{\zeta}$, the decisions of the previous $i-1$ source nodes (i.e. $\mathcal{I}_{1, k}^{\zeta}, \ldots, \mathcal{I}_{i-1, k}^{\zeta}, \forall k \in\{1,2, \ldots, K\}$ ), and its predictions of the decisions of the following $N-i$ source nodes. At the end of this stage, each source node $S_{i}$ will have determined its relay selections $\mathcal{I}_{i}^{\zeta}$, and thus, the number of source nodes $n_{r_{k}}^{\zeta}$ selecting each relay $R_{k}$ will be known to all source nodes. Two distributed best-response multi-relay selection algorithms based on constrained and unconstrained relay selections will be discussed and devised in Sections 4 and 5 , respectively.

\section{Stage 2: Data Transmission}

Stage 2 comprises the data transmission in the broadcasting and cooperation phases. Specifically, each source node broadcasts its data symbol in the broadcasting phase, and the selected relay(s) will forward the source nodes' signals in the cooperation phase. In return, each source node receives a utility $\mathcal{U}_{i, k}\left(E_{r_{k}}^{\zeta}, n_{r_{k}}^{\zeta}\right)$ according to the amount of harvested energy $\xi_{r_{k}}^{\zeta}$ at each relay $R_{k}$, and the number of source nodes $n_{r_{k}}^{\zeta}$ sharing that relay. Thus, the source nodes selecting relay $R_{k}$ in frame $\zeta$ can infer the amount of harvested energy at that relay based on the received utility. In turn, let $\phi_{i, k}^{\zeta} \sim p_{\mathcal{E}_{r_{k}}^{\zeta}}\left(\cdot \mid \theta_{r_{k}}\right)$ be the inferred information by source node $S_{i}$ about the state of relay $R_{k}$, which can collectively be expressed as

$$
\begin{aligned}
& \mathbf{p}_{\mathcal{E}_{r_{k}}^{\zeta}}\left(\Phi_{r_{k}}^{\zeta} \mid \theta_{r_{k}}\right)= \\
& \quad\left\{p_{\mathcal{E}_{r_{k}}^{\zeta}}\left(\phi_{1, k}^{\zeta} \mid \theta_{r_{k}}\right), \ldots, p_{\mathcal{E}_{r_{k}}^{\zeta}}\left(\phi_{N, k}^{\zeta} \mid \theta_{r_{k}}\right) \mid \theta_{r_{k}} \in \Theta\right\},
\end{aligned}
$$

and

$$
\begin{aligned}
& \mathbf{p}_{\mathcal{E}_{R}^{\zeta}}\left(\Phi_{R}^{\zeta} \mid \boldsymbol{\theta}\right)= \\
& \left\{\mathbf{p}_{\mathcal{E}_{r_{1}}^{\zeta}}\left(\Phi_{r_{1}}^{\zeta} \mid \theta_{r_{k}}\right), \ldots, \mathbf{p}_{\mathcal{E}_{r_{K}}^{\zeta}}\left(\Phi_{r_{K}}^{\zeta} \mid \theta_{r_{k}}\right) \mid \boldsymbol{\theta} \in \Theta^{K}\right\} .
\end{aligned}
$$

The inferred information by all source nodes about all relays' states will be used in the subsequent learning algorithm to update their beliefs about the state of each relay in each communication frame. Specifically, the inferred information $\phi_{i, k}^{\zeta} \sim p_{\mathcal{E}_{r_{k}}^{\zeta}}\left(\cdot \mid \theta_{r_{k}}\right)$ captures the amount of energy harvested by relay $R_{k}$, as observed by source $S_{i}$ in communication frame $\zeta$. It is worth-mentioning that the source nodes that did not select a relay $R_{k}$ cannot infer the quality of that relay, and thus have no inferred information.

\section{Stage 3: Non-Bayesian Learning}

In this last stage, each source node updates its belief using the inferred information from the cooperative data transmissions, and according a non-Bayesian learning (NBL) algorithm. The motivation behind the use of the NBL algorithm is to ensure that the source nodes can learn the true state of the relays. More importantly, the source nodes can utilize the inferred information to cooperatively estimate the immediate belief information about each relay state in a distributed manner ${ }^{6}$. To that end, two main steps must be performed in the learning algorithm. In the first step, each source node $S_{i}$ updates its local belief $\beta_{i, k}^{\zeta}$ of $\theta_{r_{k}}$ (i.e. $\beta_{i, k}^{\zeta}\left(\theta_{k}\right)$ ), and then shares it with the other source nodes so as to enhance the learning/convergence rate and accuracy ${ }^{7}$. Therefore, using Bayes' theorem, each source node $S_{i}$ updates its immediate belief of the state of relay $R_{k}$ as

$\beta_{i, k}^{\zeta}\left(\theta_{k}\right)=\frac{p_{\mathcal{E}_{r_{k}}^{\zeta}}\left(\phi_{i, k}^{\zeta} \mid \theta_{r_{k}}\right) p_{r_{k}}^{\zeta-1}\left(\theta_{r_{k}}\right)}{\sum_{\theta_{r_{k}} \in \Theta} p_{\mathcal{E}_{r_{k}}^{\zeta}}\left(\phi_{i, k}^{\zeta} \mid \theta_{r_{k}}\right) p_{r_{k}}^{\zeta-1}\left(\theta_{r_{k}}\right)}, \forall \theta_{r_{k}} \in \Theta$

which is revealed to all the other source nodes. Consequently, if source $S_{i}$ selects relay $R_{k}$ in frame $\zeta$ (i.e. $\mathcal{I}_{i, k}^{\zeta}=1$ ), it will incorporate its inferred information signal $\phi_{i, k}^{\zeta}$ into its immediate belief $\beta_{i, k}^{\zeta}\left(\theta_{k}\right)$ as per (25); otherwise, it will use its previous belief (i.e. when $\mathcal{I}_{i, k}^{\zeta}=0$, then $\beta_{i, k}^{\zeta}\left(\theta_{k}\right)=0$, and thus $p_{r_{k}}^{\zeta-1}\left(\theta_{r_{k}}\right)$ is used). Thus, in the second step, the immediate belief $\beta_{i, k}^{\zeta}\left(\theta_{r_{k}}\right)$ of each source $S_{i}\left(\forall \theta_{r_{k}} \in \Theta\right.$

${ }^{6}$ It is noteworthy the learning process of each relay state $\theta_{r_{k}}$ is independent of the other relays, which significantly decreases computational costs.

7 The more accurate the belief is, the better the relay selections of each source node are (i.e. better utility). 


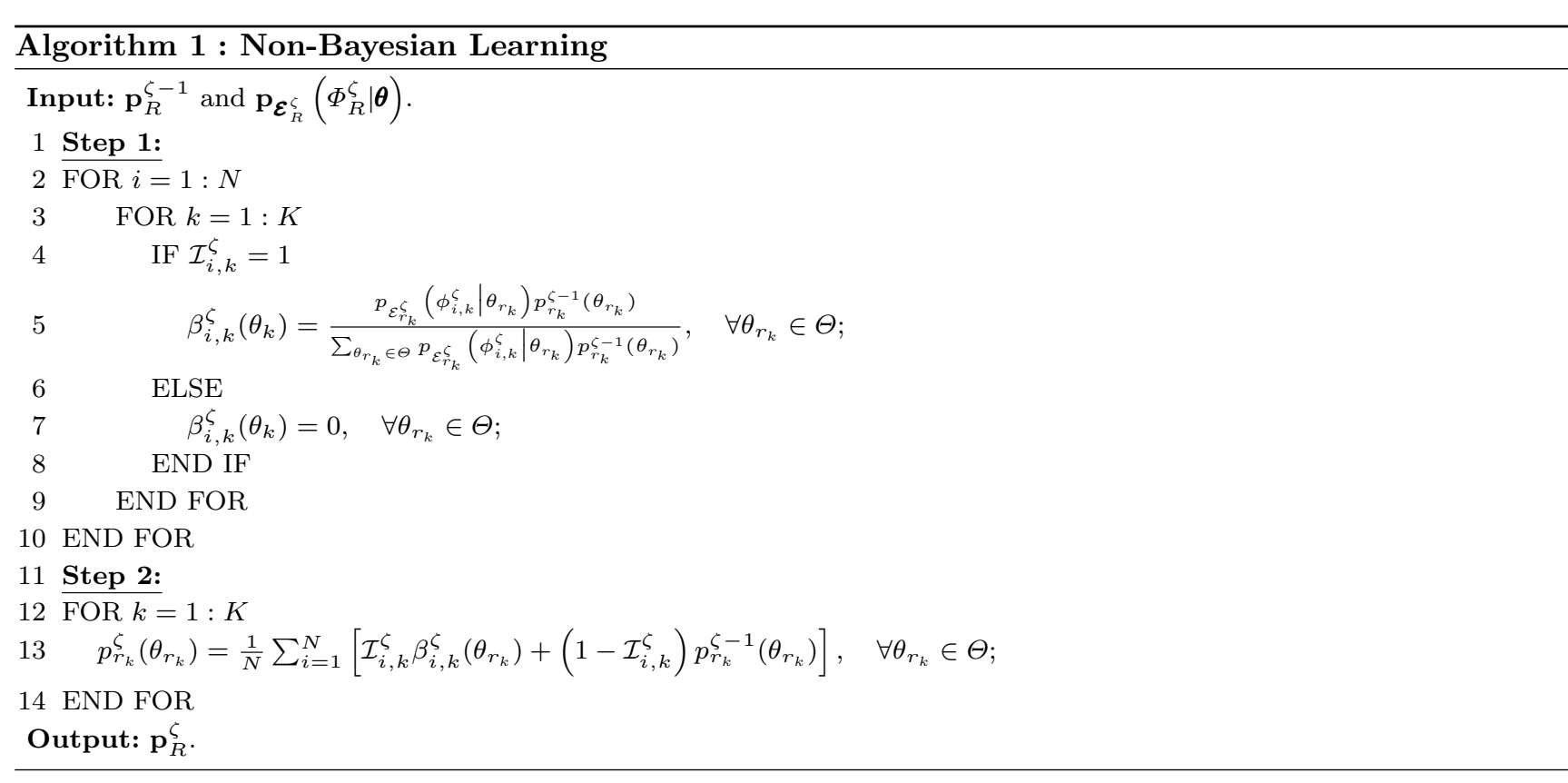

$p_{r_{k}}^{\zeta}\left(\theta_{r_{k}}\right)=p_{r_{k}}^{\zeta-1}\left(\theta_{r_{k}}\right)+\frac{1}{N} \sum_{i=1}^{N} \mathcal{I}_{i, k}^{\zeta}\left(\frac{p_{\mathcal{E}_{r_{k}}^{\zeta}}\left(\phi_{i, k}^{\zeta} \mid \theta_{r_{k}}\right)}{\Lambda\left(\phi_{i, k}^{\zeta}\right)}\right) p_{r_{k}}^{\zeta-1}\left(\theta_{r_{k}}\right), \forall \theta_{r_{k}} \in \Theta$ and $\forall k \in\{1,2, \ldots, K\}$

and $\forall k \in\{1,2, \ldots, K\})$ is linearly combined with the other source nodes' beliefs, as ${ }^{8}$

$$
p_{r_{k}}^{\zeta}\left(\theta_{r_{k}}\right)=\frac{1}{N} \sum_{i=1}^{N}\left[\mathcal{I}_{i, k}^{\zeta} \beta_{i, k}^{\zeta}\left(\theta_{r_{k}}\right)+\left(1-\mathcal{I}_{i, k}^{\zeta}\right) p_{r_{k}}^{\zeta-1}\left(\theta_{r_{k}}\right)\right]
$$

$\forall \theta_{r_{k}} \in \Theta$ and $\forall k \in\{1,2, \ldots, K\}$. Finally, the proposed NBL algorithm is outlined in Algorithm 1.

The properties of the proposed NBL algorithm can be summarized as follows.

\subsubsection{Complexity:}

The first step of the NBL algorithm requires a total of $N K$ iterations, while the second step requires $K$ iterations. Hence, the overall complexity of the NBL algorithm is $\mathcal{O}(K(N+1))$ (i.e. polynomial-time complexity), and thus can be performed efficiently.

\footnotetext{
8 The source nodes are assumed to be rational, and thus have an incentive to share their true belief information so as to improve the overall network learning. Moreover, the existence of malicious nodes in the network may lead the learning algorithm to be vulnerable to attacks; however, this is beyond the scope of this paper, and may be pursued in future work.
}

\subsubsection{Convergence:}

Note that the combined belief of each relay in communication frame $\zeta$ can be re-written as given in (27), where

$\Lambda\left(\phi_{i, k}^{\zeta}\right)=\sum_{\theta_{r_{k}} \in \Theta} p_{\mathcal{E}_{r_{k}}^{\zeta}}\left(\phi_{i, k}^{\zeta} \mid \theta_{r_{k}}\right) p_{r_{k}}^{\zeta-1}\left(\theta_{r_{k}}\right)$,

which represents the estimation of the probability distribution of $\phi_{i, k}^{\zeta}$ in communication frame $\zeta$. Thus, the convergence of the NBL algorithm can be stated in the following theorem.

Theorem 1 (Weak Convergence): Given that all source nodes update their beliefs according to (25) and $(26)$ with $p_{r_{k}}^{(0)}\left(\theta_{r_{k}}\right)>0, \forall k \in\{1,2, \ldots, K\}$ and $\forall \theta_{r_{k}} \in \Theta$, then the proposed NBL algorithm converges weakly to the true relay state $\theta_{r_{k}}^{*}$ as

$$
\begin{aligned}
\Lambda\left(\phi_{i, k}^{\zeta}\right) & =\sum_{\theta_{r_{k}} \in \Theta} p_{\mathcal{E}_{r_{k}}^{\zeta}}\left(\phi_{i, k}^{\zeta} \mid \theta_{r_{k}}\right) p_{r_{k}}^{\zeta-1}\left(\theta_{r_{k}}\right) \\
& \rightarrow p_{\mathcal{E}_{r_{k}}^{\zeta}}\left(\phi_{i, k}^{\zeta} \mid \theta_{r_{k}}^{*}\right)
\end{aligned}
$$

as $\zeta \rightarrow \infty, \forall \phi_{i, k}^{\zeta} \in \Theta$.

Proof See proof in [21].

Remark 9: It is noteworthy that as soon as the NBL algorithm converges to the true priors and beliefs, it need not be executed any further, provided that the 
$\left.\mathbb{U}_{i, k}\right|_{\mathcal{I}_{i}^{\zeta}=\boldsymbol{\omega}_{q}}=\omega_{q, k} \sum_{\theta_{r_{k}} \in \Theta} \sum_{\xi=0}^{\infty} \mathcal{U}_{i, k}\left(\xi, n_{s_{i}, r_{k}}^{\zeta}+\left.\mathcal{J}_{i, k}^{\zeta}\right|_{\mathcal{I}_{i}^{\zeta}=\boldsymbol{\omega}_{q}}+\omega_{q, k}\right) p_{\xi_{r_{k}}^{\zeta}}\left(\xi \mid \theta_{r_{k}}\right) p_{r_{k}}^{\zeta}\left(\theta_{r_{k}}\right)$

relays' states remain the same during the entire network operation. This in turn significantly reduces communications overheads.

\section{Distributed Relay Selection Game with Constrained Selections}

In this Section, it is assumed that each source node can select a maximum of $\kappa \leq K$ relays. This could be due to several technical reasons; for instance, imperfect timing synchronization, excessive communication overheads and delays, etc., in which case it is imperative that the number of selected relays be constrained. Now, let $\mathbf{n}_{-i}^{\zeta}=\left[n_{-i, 1}^{\zeta}, n_{-i, 2}^{\zeta}, \ldots, n_{-i, K}^{\zeta}\right]$, where

$n_{-i, k}^{\zeta}=\sum_{j=1, j \neq i}^{N} \mathcal{I}_{j, k}^{\zeta}$

denotes the total number of source nodes - except for source node $S_{i}$ - selecting relay $R_{k}$. Hence, the bestresponse with constrained selections (BR-CS) optimization problem of each source $S_{i}$ is expressed as

\section{BR-CS:}

$$
\begin{aligned}
& \mathcal{I}_{i}^{\zeta, *}\left(\mathbf{p}_{R}^{\zeta}, \mathbf{n}_{-i}^{\zeta}\right)=\underset{\mathcal{I}_{i, k}^{\zeta} \in\{0,1\}, \forall k}{\operatorname{argmax}} \sum_{k=1}^{K} \mathcal{I}_{i, k}^{\zeta} \cdot \mathbb{U}_{i, k}\left(\mathbf{p}_{r_{k}}^{\zeta}, n_{-i, k}^{\zeta}\right) \\
& \text { s.t. } \quad \sum_{k=1}^{K} \mathcal{I}_{i, k}^{\zeta} \leq \kappa,
\end{aligned}
$$

where $\mathbb{U}_{i, k}\left(\mathbf{p}_{r_{k}}^{\zeta}, n_{-i, k}^{\zeta}\right)$ is the expected utility, as

$$
\begin{aligned}
& \mathbb{U}_{i, k}\left(\mathbf{p}_{r_{k}}^{\zeta}, n_{-i, k}^{\zeta}\right)= \\
& \sum_{\theta_{r_{k}} \in \Theta} \sum_{\xi=0}^{\infty} \mathcal{U}_{i, k}\left(\xi, n_{-i, k}^{\zeta}+\mathcal{I}_{i, k}^{\zeta}\right) p_{\xi_{r_{k}}}\left(\xi \mid \theta_{r_{k}}\right) p_{r_{k}}^{\zeta}\left(\theta_{r_{k}}\right),
\end{aligned}
$$

where $p_{\xi_{r_{k}}^{\zeta}}\left(\xi \mid \theta_{r_{k}}\right)$ is the conditional PDF on the relay state $\theta_{r_{k}} \in \Theta$. Clearly, the selection of relay $R_{k}$ also depends on all the other relays. In turn, the best-response of source $S_{i}$ can be obtained by determining all possible combinations of the $\kappa$ relays out of the total number of relays $K$. Now, let $\boldsymbol{\Omega}=\left\{\boldsymbol{\omega}_{1}, \boldsymbol{\omega}_{2}, \ldots, \boldsymbol{\omega}_{Q}\right\}$ be the set of all combinations of $q$ (for $1 \leq q \leq \kappa$ ) relays out of $K$ relays, with the total number of relay combinations being defined as

$Q(\kappa, K) \triangleq \sum_{q=1}^{\kappa}\left(\begin{array}{c}K \\ q\end{array}\right)$ and $\left(\begin{array}{c}K \\ q\end{array}\right)=\frac{K !}{q !(K-q) !}$ is the binomial coefficient. Moreover, $\boldsymbol{\omega}_{q}=\left[\omega_{q, 1}, \omega_{q, 2}, \ldots, \omega_{q, K}\right]$ is one of the possible combinations, such that $\omega_{q, k} \in\{0,1\}$ indicates whether relay $R_{k}$ is selected. On the other hand, let

$\mathbf{n}_{s_{i}}^{\zeta}=\left[n_{s_{i}, r_{1}}^{\zeta}, n_{s_{i}, r_{2}}^{\zeta}, \ldots, n_{s_{i}, r_{K}}^{\zeta}\right]$,

where $n_{s_{i}, r_{k}}^{\zeta} \triangleq \sum_{j=1}^{i-1} \mathcal{I}_{i, k}^{\zeta}$ denotes the number of source nodes choosing relay $R_{k}$ before source $S_{i}$. Now, let $\mathcal{J}_{i}^{\zeta} \triangleq$ $\left[\mathcal{J}_{i, 1}^{\zeta}, \mathcal{J}_{i, 2}^{\zeta}, \ldots, \mathcal{J}_{i, K}^{\zeta}\right]$ be the selection decisions of the source nodes after source $S_{i}$ of all relays, which can be expressed as $\mathcal{J}_{i}^{\zeta}=\mathcal{J}_{i+1}^{\zeta}+\boldsymbol{\mathcal { I }}_{i+1}^{\zeta}$. Moreover, let $\left.\mathcal{J}_{i, k}^{\zeta}\right|_{\mathcal{I}_{i}^{\zeta}=\boldsymbol{\omega}_{q}}$ be the expected number of subsequent source nodes who will select relay $R_{k}$ when $\boldsymbol{I}_{i}^{\zeta}=\boldsymbol{\omega}_{q}$, for $\boldsymbol{\omega}_{q} \in \boldsymbol{\Omega}$. In turn, the expected number of source nodes - excluding source $S_{i}$ - choosing different relays is given by

$\left.\overline{\mathbf{n}}_{-i}^{\zeta}\right|_{\boldsymbol{I}_{i}^{\zeta}=\boldsymbol{\omega}_{q}}=\mathbf{n}_{s_{i}}^{\zeta}+\left.\mathcal{J}_{i}^{\zeta}\right|_{\mathcal{I}_{i}^{\zeta}=\boldsymbol{\omega}_{q}}$

The expected utility of source $S_{i}$ selecting $R_{k}$-conditional on $\mathcal{I}_{i}^{\zeta}=\boldsymbol{\omega}_{q}$-is alternatively determined as given in (36). Thus, the expected sum-utility of source $S_{i}$, conditional on $\mathcal{I}_{i}^{\zeta}=\boldsymbol{\omega}_{q}$, is obtained as

$\left.\mathbb{U}_{i}\right|_{\boldsymbol{I}_{i}^{\zeta}=\boldsymbol{\omega}_{q}}=\left.\sum_{k=1}^{K} \mathbb{U}_{i, k}\right|_{\boldsymbol{I}_{i}^{\zeta}=\boldsymbol{\omega}_{q}}$.

Hence, the best-response strategy of source $S_{i}$ is determined by finding the optimal $\boldsymbol{\omega}_{q}$ which maximizes the expected utility $\left.\mathbb{U}_{i}\right|_{\mathcal{I}_{i}^{\zeta}=\boldsymbol{\omega}_{q}}$ as

$\boldsymbol{\omega}^{*}=\left.\underset{\boldsymbol{\omega}_{q} \in \boldsymbol{\Omega}}{\operatorname{argmax}} \mathbb{U}_{i}\right|_{\boldsymbol{I}_{i}^{\zeta}=\boldsymbol{\omega}_{q}}$.

In summary, each source $S_{i}$ must calculate its expected utility $\left.\mathbb{U}_{i, k}\right|_{\mathcal{I}_{i}^{\zeta}=\boldsymbol{\omega}_{q}}$ for selecting each relay by predicting the expected number of subsequent source nodes selecting relay $R_{k}$ when $\mathcal{I}_{i}^{\zeta}=\boldsymbol{\omega}_{q}$. After that, the sum-utility $\left.\mathbb{U}_{i}\right|_{\boldsymbol{I}_{i}^{\zeta}=\boldsymbol{\omega}_{q}}$ of source $S_{i}$ over all $K$ relays is calculated for each $\mathcal{I}_{i}^{\zeta}=\omega_{q}$. Lastly, the combination $\omega_{q} \in \Omega$ that maximizes the sum-utility of source $S_{i}$ is selected. It should be noted that the $N^{t h}$ source node $S_{N}$ does not have to predict the number of subsequent source nodes selecting each relay, since it is the last source node and has already observed all previous source nodes' decisions. As for the other source nodes $S_{i}($ for $i \neq N)$, they must recursively predict the following source nodes' decisions, and then make their decisions based on the predictions and current observations.

Definition 1 (Relay Selection Game with Constrained Selections (RSG-CS)): A relay selection 


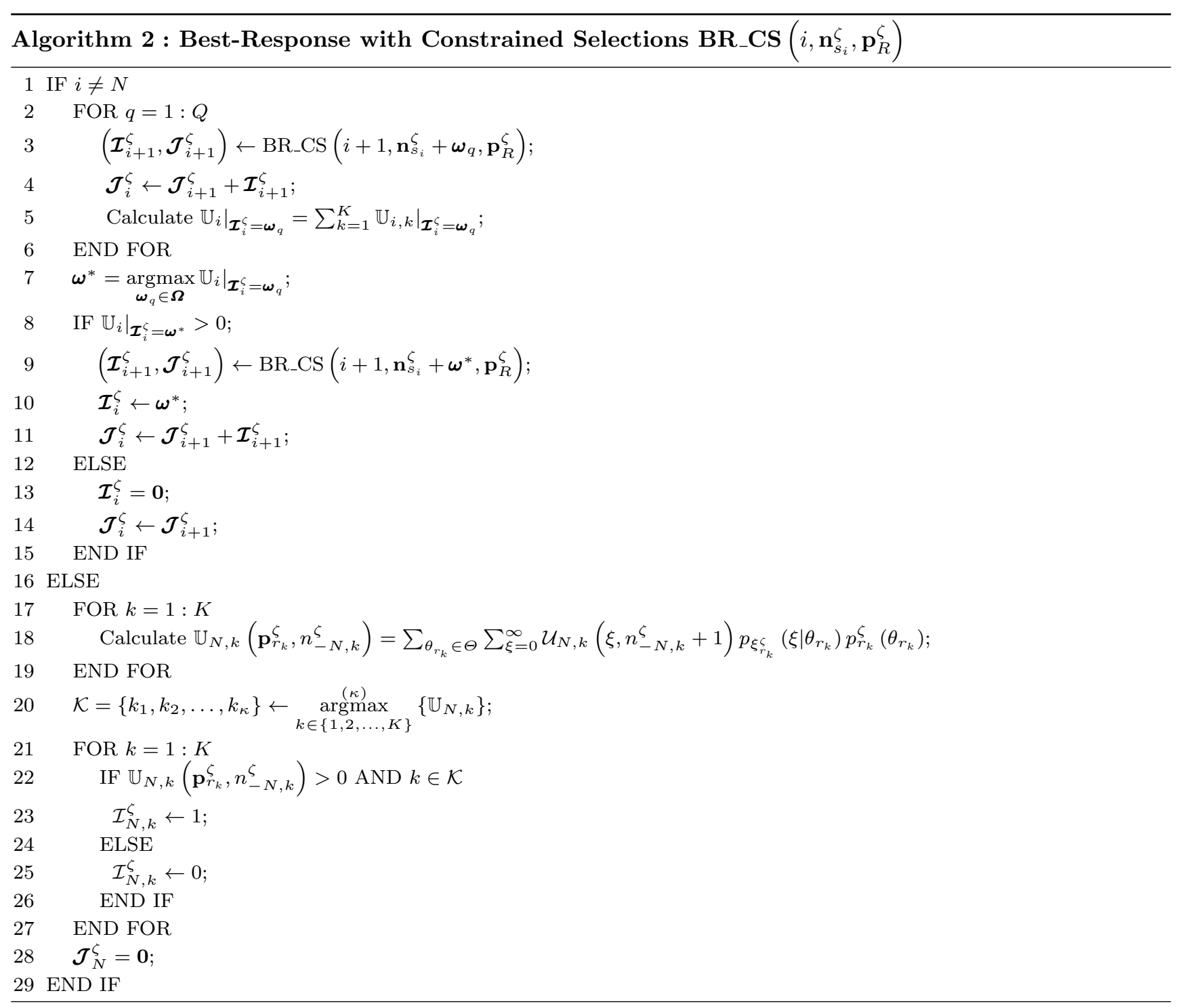

game with constrained selections (RSG-CS) is defined in terms of the $N$ source nodes and $K$ relays involved in the relay selection game with $\kappa$ selections in each communication frame $\zeta$, which is based on: (i) each source node $S_{i}$ (for $i \in\{1,2, \ldots, N\}$ ) sequentially selecting relays, (ii) number of source nodes $\mathbf{n}_{s_{i}}^{\zeta}$ selecting each relay before source $S_{i}$, and (iii) beliefs about all $K$ relays' states $\mathbf{p}_{R}^{\zeta}$.

Definition 2 (Relay Selection Profile (RSP)): Let $\mathcal{I}^{\zeta}=\left[\mathcal{I}_{1}^{\zeta}, \mathcal{I}_{2}^{\zeta}, \ldots, \mathcal{I}_{N}^{\zeta}\right]$ be defined as the relay selection profile (RSP), where $\mathcal{I}_{i}^{\zeta}$ is the set of relays selected by source $S_{i}, \forall i \in\{1,2, \ldots, N\}$.

The RSG-CS is performed by applying the bestresponse with constrained selections BR_CS $\left(i, \mathbf{n}_{s_{i}}^{\zeta}, \mathbf{p}_{R}^{\zeta}\right)$ recursive algorithm sequentially per source $S_{i}$ (for $i \in$ $\{1,2, \ldots, N\})$, as given in Algorithm 2. Consequently, the RSP $\mathcal{I}^{\zeta, *}=\left[\mathcal{I}_{1}^{\zeta, *}, \mathcal{I}_{2}^{\zeta, *}, \ldots, \boldsymbol{I}_{N}^{\zeta, *}\right]$, and correspondingly, the number of source nodes selecting each relay, $\mathbf{n}_{R}^{\zeta, *}=\left[n_{r_{1}}^{\zeta, *}, n_{r_{2}}^{\zeta, *}, \ldots, n_{r_{K}}^{\zeta, *}\right]$ are obtained. It should be noted that in Algorithm 2, $\operatorname{argmax}^{(\kappa)}\{\cdot\}$ indicates the $\kappa$ highest values.

In the following subsections, the properties of the proposed relay selection game with constrained selections are discussed; however, a few definitions must first be stated.

Definition 3 (Nash Equilibrium (NE)): Given the relay's states beliefs $\mathbf{p}_{R}^{\zeta}$, the $\operatorname{RSP} \mathcal{I}^{\zeta, *}=\left[\boldsymbol{I}_{1}^{\zeta, *}, \boldsymbol{I}_{2}^{\zeta, *}, \ldots, \mathcal{I}_{N}^{\zeta, *}\right]$ is a Nash Equilibrium (NE) of the RSG-CS if and only if every source node $S_{i}$ performs its best-response relay selection, such that its expected utility is maximized, when all the other source nodes perform their bestresponse relay selections as well. 
$\mathbb{U}_{i, k}\left(\mathbf{p}_{r_{k}}^{\zeta}, n_{s_{i}, r_{k}}^{\zeta}\right)=\sum_{\theta_{r_{k}} \in \Theta} \sum_{\xi=0}^{\infty} \mathcal{U}_{i, k}\left(\xi, n_{s_{i}, r_{k}}^{\zeta}+\left.\mathcal{J}_{i, k}^{\zeta}\right|_{\mathcal{L}_{i, k}^{\zeta}=1}+1\right) p_{\xi_{r_{k}}^{\zeta}}\left(\xi \mid \theta_{r_{k}}\right) p_{r_{k}}^{\zeta}\left(\theta_{r_{k}}\right)$

Definition 4 (Subgame Perfect Nash Equilibrium): $\mathrm{A} \mathrm{NE}$ is subgame perfect if and only if it is a NE for every subgame [35].

\subsection{Subgame Perfect Nash Equilibrium for RSG-CS}

Based on the above definitions, the RSP obtained via the BR_CS $\left(i, \mathbf{n}_{s_{i}}^{\zeta}, \mathbf{P}_{R}^{\zeta}\right)$ algorithm is a subgame perfect NE of the RSG-CS according to the following theorem.

Theorem 2 (Subgame Perfect Nash Equilibrium for RSG-CS): Based on the relays' states beliefs $\mathbf{p}_{R}^{\zeta}$, the equilibrium $\operatorname{RSP} \mathcal{I}^{\zeta, *}=\left[\mathcal{I}_{1}^{\zeta, *}, \mathcal{I}_{2}^{\zeta, *}, \ldots, \boldsymbol{I}_{N}^{\zeta, *}\right]$, where $\boldsymbol{I}_{i}^{\zeta, *}=$ BR_CS $\left(i, \mathbf{n}_{s_{i}}^{\zeta}, \mathbf{p}_{R}^{\zeta}\right), \forall i \in\{1,2, \ldots, N\}$, is subgame perfect NE for the RSG-CS.

Proof This theorem can be proved by contradiction. Consider the relay selection profile $\boldsymbol{\mathcal { I }}_{i}^{\zeta}$ of each source node $S_{i}, \forall i \in\{1,2, \ldots, N\}$, and let $\mathcal{I}_{i}^{\zeta,{ }^{\prime}}$ be a set of relay selection decisions by source $S_{i}$ which is different from the best-response relay selection decisions $\mathcal{I}_{i}^{\zeta, *}$. Then, as per (31) and (38), $\left.\mathbb{U}_{i}\right|_{\mathcal{I}_{i}^{\zeta}=\mathcal{I}_{i}^{\zeta,}}<\left.\mathbb{U}_{i}\right|_{\mathcal{I}_{i}^{\zeta}=\mathcal{I}_{i}^{\zeta, *}}$, and hence $\boldsymbol{I}_{i}^{\zeta,}$ cannot be the best-response relay selection profile, in which case source node $S_{i}$ must deviate to $\mathcal{I}_{i}^{\zeta, *}$. Thus, the RSP $\mathcal{I}^{\zeta, *}$ obtained from BR_CS $\left(i, \mathbf{n}_{s_{i}}^{\zeta}, \mathbf{P}_{R}^{\zeta}\right)$ must be the NE for the subgame of each source node $S_{i}$ (for $i \in\{1,2, \ldots, N\})$. Consequently, the RSP $\mathcal{I}^{\zeta, *}$ is subgame perfect NE for the RSG-CS.

\subsection{Complexity of RSG-CS}

To quantify the complexity of the RSG-CS, the total number of calls to the BR_CS algorithm must be determined. First, consider source node $S_{1}$, which requires $(Q(\kappa, K))^{N-1}$ iterations to determine its bestresponse relay selections in response to the predicted selections of subsequent source nodes. This is due to the recursive structure of the BR_CS $\left(i, \mathbf{n}_{s_{i}}^{\zeta}, \mathbf{p}_{R}^{\zeta}\right)$ algorithm. Moreover, the following source nodes require less iterations to determine their best-response relay selections. On the other hand, the $N^{t h}$ source node $S_{N}$ performs one call to the BR_CS algorithm as it is the last source node, and thus has observed the relay selections of the $N-1$ previous source nodes. It can be verified that $Q(K, K)=2^{K}-1$, and thus $Q(\kappa, K)=$ $2^{K}-1-\sum_{q=\kappa+1}^{K}\left(\begin{array}{c}K \\ q\end{array}\right)<2^{K}-1, \forall \kappa \in\{1,2, \ldots, K-1\}$. In turn, the overall complexity of the RSG-CS is determined as $\mathcal{O}\left(2^{K(N-1)}\right)$, which forms the worst-case complexity bound of the BR_CS algorithm. Clearly, the smaller the value of $\kappa$ is, the lesser the complexity of the RSG-CS.

\section{Distributed Relay Selection Game with Unconstrained Selections}

In the unconstrained selections scenario, the source nodes can select all possible relays (i.e. $\kappa=K$ ). Consequently, the best-response with unconstrained selections (BR-US) optimization problem of each source $S_{i}$ can be expressed as

\section{BR-US:}

$\boldsymbol{I}_{i}^{\zeta, *}\left(\mathbf{p}_{R}^{\zeta}, \mathbf{n}_{-i}^{\zeta}\right)=\underset{\mathcal{I}_{i, k}^{\zeta} \in\{0,1\}, \forall k}{\operatorname{argmax}} \sum_{k=1}^{K} \mathcal{I}_{i, k}^{\zeta} \cdot \mathbb{U}_{i, k}\left(\mathbf{p}_{r_{k}}^{\zeta}, n_{-i, k}^{\zeta}\right)$,

where $\mathbb{U}_{i, k}\left(\mathbf{p}_{r_{k}}^{\zeta}, n_{-i, k}^{\zeta}\right)$ is as defined in (32). Since there is no constraint on the number of selected relays, the above optimization problem can be reformulated as

\section{BR-US:}

$$
\mathcal{I}_{i}^{\zeta, *}\left(\mathbf{p}_{R}^{\zeta}, \mathbf{n}_{-i}^{\zeta}\right)=\sum_{k=1}^{K} \underset{\mathcal{I}_{i, k}^{\zeta} \in\{0,1\}}{\operatorname{argmax}} \mathcal{I}_{i, k}^{\zeta} \cdot \mathbb{U}_{i, k}\left(\mathbf{p}_{r_{k}}^{\zeta}, n_{-i, k}^{\zeta}\right) .
$$

That is, each source can select as many relays as possible, provided that the selected relay yields positive utility, independently of the other source nodes' selections. Thus, each source $S_{i}$ can select a relay $R_{k}$ according to

$$
\begin{aligned}
\mathcal{I}_{i, k}^{\zeta, *} & =\underset{\mathcal{I}_{i, k}^{\zeta} \in\{0,1\}}{\operatorname{argmax}} \mathcal{I}_{i, k}^{\zeta} \cdot \mathbb{U}_{i, k}\left(\mathbf{p}_{r_{k}}^{\zeta}, n_{-i, k}^{\zeta}\right) \\
& = \begin{cases}1, & \text { if } \mathbb{U}_{i, k}\left(\mathbf{p}_{r_{k}}^{\zeta}, n_{-i, k}^{\zeta}\right)>0, \\
0, & \text { otherwise. }\end{cases}
\end{aligned}
$$

As before, since the source nodes make their relay selection decisions sequentially, a source node $S_{i}$ does not know the decisions of the following source nodes, and thus must predict them. Based on (30), (34) and (35), the estimated number of source nodes choosing a relay $R_{k}$ excluding source node $S_{i}$ is given by

$\left.n_{-i, k}^{\zeta}\right|_{\mathcal{I}_{i, k}^{\zeta}}=n_{s_{i}, r_{k}}^{\zeta}+\left.\mathcal{J}_{i, k}^{\zeta}\right|_{\mathcal{I}_{i, k}^{\zeta}}$

Similarly to (32), the expected utility of source $S_{i}$ when it selects relay $R_{k}$ (i.e. $\mathcal{I}_{i, k}^{\zeta}=1$ ) is given by (43). Consequently, the best-response relay selection of source node $S_{i}$ is determined as 


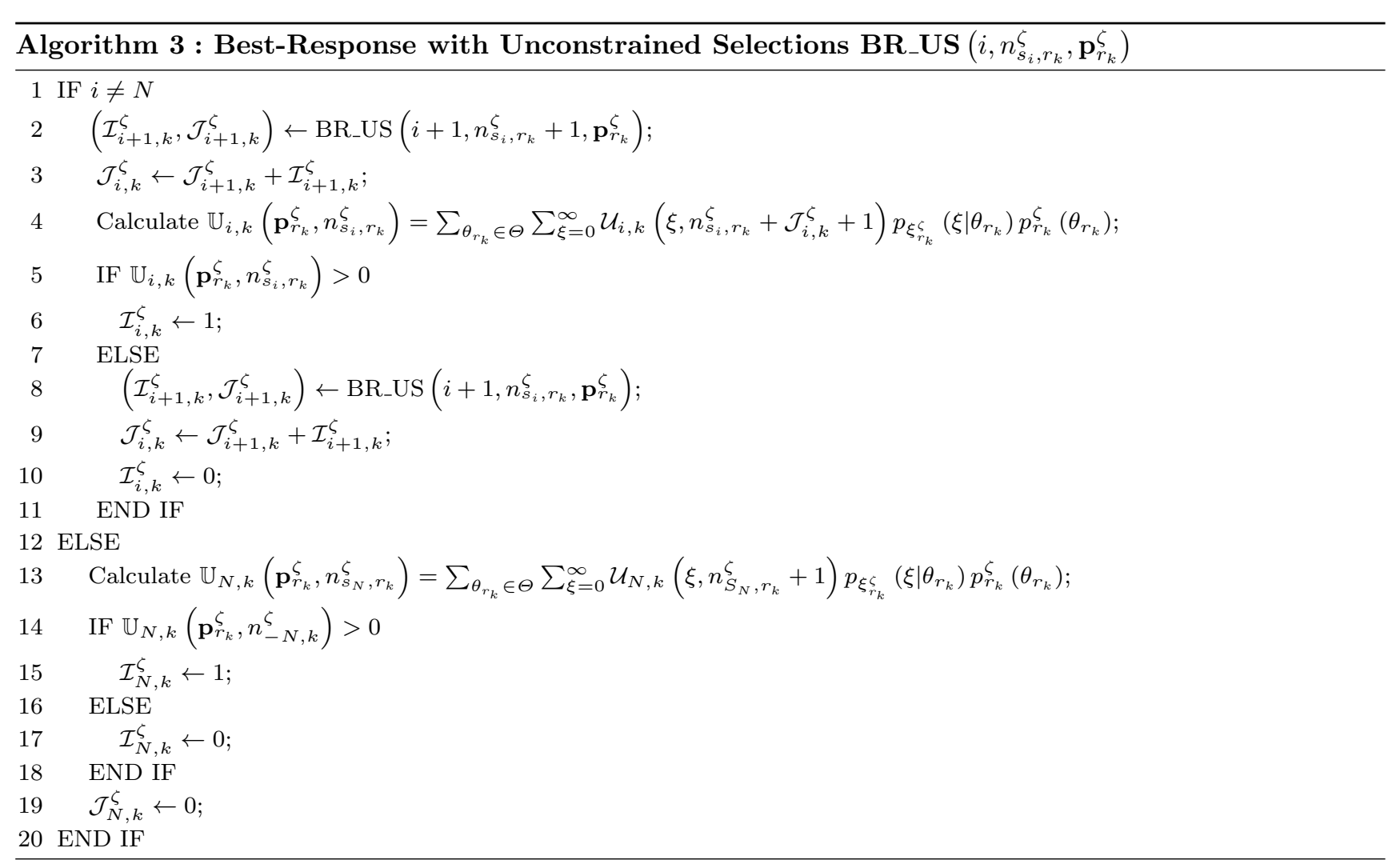

$\mathcal{I}_{i, k}^{\zeta, *}= \begin{cases}1, & \text { if } \mathbb{U}_{i, k}\left(\mathbf{p}_{r_{k}}^{\zeta}, n_{s_{i}, r_{k}}^{\zeta}\right)>0, \\ 0, & \text { otherwise. }\end{cases}$

Definition 5 (Relay Selection Game with Unconstrained Selections (RSG-US)): A relay selection game with unconstrained selections (RSG-US) is defined in terms of the $N$ source nodes and $K$ relays involved in the relay selection game where all $N$ source nodes can select up to $K$ relays in each communication frame $\zeta$, which is based on: (i) each source node $S_{i}$ (for $i \in\{1,2, \ldots, N\})$ sequentially selecting each relay $R_{k}$ (for $k \in\{1,2, \ldots, K\}$ ), (ii) source nodes $n_{s_{i}, r_{k}}^{\zeta}$ selecting each relay $R_{k}$ before source $S_{i}$, and (iii) beliefs about each relay $R_{k}$ 's states $\boldsymbol{p}_{r_{k}}^{\zeta}, \forall k \in\{1,2, \ldots, K\}$.

This implies that in the RSG-US, for source $S_{i}$ to predict $\left.\mathcal{J}_{i, k}^{\zeta}\right|_{\mathcal{I}_{i, k}^{\zeta}=1}$, it must predict the decisions of the following source nodes (i.e. $S_{i+1}$ to $S_{N}$ ), and then update $\mathcal{J}_{i, k}^{\zeta}=\mathcal{J}_{i+1, k}^{\zeta}+\mathcal{I}_{i+1, k}^{\zeta}$. As for the last source node $S_{N}$, it does not need to do any prediction as it knows the decisions of all previous $N-1$ source nodes, and thus $\mathcal{J}_{N, k}^{\zeta}=0$. The best-response with unconstrained selections is performed on each relay $R_{k}, \forall k \in\{1,2, \ldots, K\}$, according to the BR_US $\left(i, n_{s_{i}, r_{k}}^{\zeta}, \mathbf{p}_{r_{k}}^{\zeta}\right)$ recursive algorithm, which is outlined in Algorithm 3.

The properties of Algorithm 3 are discussed in the following two subsections.

\subsection{Subgame Perfect Nash Equilibrium for RSG-US}

As before, the RSP obtained via the BR_US $\left(i, n_{s_{i}, r_{k}}^{\zeta}, \mathbf{p}_{r_{k}}^{\zeta}\right)$ algorithm is a subgame perfect NE of the RSG-US, as per the following theorem.

Theorem 3 (Subgame Perfect Nash Equilibrium for RSG-US): Given relays's states beliefs $\mathbf{p}_{R}^{\zeta}$, the equilibrium $\operatorname{RSP} \boldsymbol{I}^{\zeta, *}=\left[\boldsymbol{I}_{1}^{\zeta, *}, \boldsymbol{I}_{2}^{\zeta, *}, \ldots, \boldsymbol{I}_{N}^{\zeta, *}\right]$, where $\mathcal{I}_{i}^{\zeta, *}=\operatorname{BR} \_\mathrm{US}\left(i, n_{s_{i}, r_{k}}^{\zeta}, \mathbf{p}_{r_{k}}^{\zeta}\right), \forall i \in\{1,2, \ldots, N\}$, is subgame perfect NE for the RSG-US, $\forall k \in\{1,2, \ldots, K\}$.

Proof As before, consider the relay selection $\mathcal{I}_{i, k}^{\zeta}$ of each source node $S_{i}, \forall i \neq N$ of relay $R_{k}$ for $k \in\{1,2, \ldots, K\}$, and let $\mathcal{I}_{i, k}^{\zeta,}$ denote a selection decision of source $S_{i}$ for relay $R_{k}$ that is different from the best-response selection decision $\mathcal{I}_{i, k}^{\zeta, *}$. Now, if $\mathcal{I}_{i, k}^{\zeta,}=0$ (while $\mathcal{I}_{i, k}^{\zeta, *}=$ 1), then $\mathbb{U}_{i, k}\left(\mathbf{p}_{r_{k}}^{\zeta}, n_{s_{i}, r_{k}}^{\zeta}\right)_{\mathcal{I}_{i, k}^{\zeta,}=0}=0$, which is less than $\mathbb{U}_{i, k}\left(\mathbf{p}_{r_{k}}^{\zeta}, n_{s_{i}, r_{k}}^{\zeta}\right)_{\mathcal{I}_{i, k}^{\zeta, *}=1}>0$, since it is not the bestresponse selection, and thus source $S_{i}$ has an incentive to deviate and make the selection decision $\mathcal{I}_{i, k}^{\zeta, *}=1$ to achieve positive utility. Similarly, if $\mathcal{I}_{i, k}^{\zeta,}=1$ (while $\left.\mathcal{I}_{i, k}^{\zeta, *}=0\right)$, then $\mathbb{U}_{i, k}\left(\mathbf{p}_{r_{k}}^{\zeta}, n_{s_{i}, r_{k}}^{\zeta}\right)_{\mathcal{I}_{i, k}^{\zeta,}=1}<0$, where as $\mathbb{U}_{i, k}\left(\mathbf{p}_{r_{k}}^{\zeta}, n_{s_{i}, r_{k}}^{\zeta}\right)_{\mathcal{I}_{i, k}^{\zeta, *}=0}=0$, which implies that source $S_{i}$ must deviate from $\mathcal{I}_{i, k}^{\zeta,}=1$ to avoid the negative utility. That is, either case yields a contradiction, which im- 
$\Delta \mathcal{R}_{i}\left(\mathbf{E}_{R}^{\zeta}, \mathbf{n}_{R}^{\zeta}\right)=\frac{1}{N+1}\left[\log _{2}\left(1+\frac{E_{B_{i}}^{\zeta}\left|h_{i, d}^{\zeta}\right|^{2}}{N_{0}}+\sum_{k=1}^{K} \mathcal{I}_{i, k}^{\zeta} \frac{E_{B_{i}}^{\zeta}\left(\frac{E_{r_{k}}^{\zeta}}{n_{r_{k}}^{\zeta}}\right)\left|h_{i, k}^{\zeta}\right|^{2}\left|h_{k, d}^{\zeta}\right|^{2}}{N_{0} \varrho_{N}\left(E_{B_{i}}^{\zeta}\left|h_{i, k}^{\zeta}\right|^{2}+\left(\frac{E_{r_{k}}^{\zeta}}{n_{r_{k}}}\right)\left|h_{k, d}^{\zeta}\right|^{2}+N_{0}\right)}\right)-\log _{2}\left(1+\frac{E_{B_{i}}^{\zeta}\left|h_{i, d}^{\zeta}\right|^{2}}{N_{0}}\right)\right]$

plies that $\mathcal{I}_{i, k}^{\zeta, *}$ is the best-response relay selection, as per BR_US $\left(i, n_{s_{i}, r_{k}}^{\zeta}, \mathbf{p}_{r_{k}}^{\zeta}\right)$. As for source node $S_{N}, \mathcal{I}_{N, k}^{\zeta, *}=1$ if relay $R_{k}$ yields a positive utility (as per (44)), and $\mathcal{I}_{N, k}^{\zeta, *}=0$, otherwise. Hence, for source node $S_{N}$, the relay selection obtained via BR_US $\left(N, n_{s_{N}, r_{k}}^{\zeta}, \mathbf{p}_{r_{k}}^{\zeta}\right)$ yields the best-response selection for relay $R_{k}$. Consequently, the RSP $\mathcal{I}^{\zeta, *}$ obtained via BR_US $\left(i, n_{s_{i}, r_{k}}^{\zeta}, \mathbf{p}_{r_{k}}^{\zeta}\right)(\forall i \in$ $\{1,2, \ldots, N\}$ and $\forall k \in\{1,2, \ldots, K\})$ is subgame perfect NE for RSG-US.

\subsection{Complexity of RSG-US}

Based on Algorithm 3, and for each relay $R_{k}$, each source node $S_{i}, \forall i \neq N$, performs $2(N-i)+1$ calls to the BR_US algorithm, which sums to $\sum_{i=1}^{N-1}(2(N-i)+1)=$ $\frac{1}{2}\left(5 N^{2}-N-1\right)$. On the other hand, source node $S_{N}$ performs only one call to the BR_US algorithm. Therefore, for selection decisions per relay requires a complexity order of $\mathcal{O}\left(N^{2}\right)$, in agreement with [21]. Hence, the overall worst-case complexity bound for the RSGUS for all $K$ relays is $\mathcal{O}\left(K N^{2}\right)$.

Remark 10: The relay selections of the BR-CS algorithm are equivalent to its BR-US algorithms when $\kappa=K$; however, the complexity of the former algorithm is still significantly higher since it must consider all possible relay combinations $\Omega, \forall \kappa \in\{1,2, \ldots, K\}$.

Lastly, it is worth-mentioning that due to the sequential nature of the proposed BR-CS and BR-US algorithms, the resulting relay selections may not necessarily be unique, as they are source nodes' order dependent. In other words, changing the order of sequential relay selection by the source nodes may yield different relay selection outcomes of both algorithms. Additionally, the resulting relay selections are not necessarily socially-efficient [35]. This is due to the fact that the resulting relay selections from any of the algorithms (and as a consequence of dependency on source nodes' order) may not necessarily maximize the social welfare (i.e. sum-of-utilities).

\section{Centralized Multi-Relay Selection}

The centralized multi-relay selection (C-MRS) problem in each communication frame $\zeta$ subject to a maximum number of relay selections $\kappa$ per source node can be formulated as a mixed integer non-linear programming (MINLP) problem as

\section{C-MRS:}

$$
\begin{aligned}
\max & \sum_{i=1}^{N}\left(\Delta \mathcal{R}_{i}\left(\mathbf{E}_{R}^{\zeta}, \mathbf{n}_{R}^{\zeta}\right)-\mathcal{P}_{i}\left(\mathbf{E}_{R}^{\zeta}, \mathbf{n}_{R}^{\zeta}\right)\right) \\
\text { s.t. } & \sum_{k=1}^{K} \mathcal{I}_{i, k}^{\zeta} \leq \kappa, \quad \forall i \in\{1,2, \ldots, N\}, \\
& \sum_{i=1}^{N} \mathcal{I}_{i, k}^{\zeta}=n_{r_{k}}^{\zeta}, \quad \forall k \in\{1,2, \ldots, K\}, \quad \text { (45a) } \\
& \mathcal{I}_{i, k}^{\zeta} \in\{0,1\}, \\
& \forall i \in\{1,2, \ldots, N\} \text { and } \forall k \in\{1,2, \ldots, K\},
\end{aligned}
$$

$$
n_{r_{k}}^{\zeta} \in\{0,1, \ldots, N\}, \quad \forall k \in\{1,2, \ldots, K\},
$$

where $\Delta \mathcal{R}_{i}\left(\mathbf{E}_{R}^{\zeta}, \mathbf{n}_{R}^{\zeta}\right)$ is given by (46). Furthermore, $\mathcal{P}_{i}\left(\mathbf{E}_{R}^{\zeta}, \mathbf{n}_{R}^{\zeta}\right)$ is the sum-payment by source $S_{i}$, as

$\mathcal{P}_{i}\left(\mathbf{E}_{R}^{\zeta}, \mathbf{n}_{R}^{\zeta}\right)=\sum_{k=1}^{K} \mathcal{I}_{i, k}^{\zeta} \cdot \mathcal{P}_{i, k}\left(E_{r_{k}}^{\zeta}, n_{r_{k}}^{\zeta}\right)$,

where $\mathcal{P}_{i, k}\left(E_{r_{k}}^{\zeta}, n_{r_{k}}^{\zeta}\right)$ is given in $(22)$. In C-MRS, the first constraint defines the maximum number of relays $\kappa$ that can be selected by each source node, while the second constraint defines the number of source nodes selecting relay $R_{k}$. The last two constraints define the range of values each decision variable takes.

Remark 11: If $\kappa<K$, then the C-MRS admits a relay selection problem with constrained selections. On the other hand, if $\kappa=K$, then each source can select as many relays as possible (i.e. unconstrained selections).

Remark 12: The C-MRS problem does not take into account the updated beliefs about each relay state, and solely determines the optimal relay selection based on the energy arrivals, and the maximum number of relay selections $\kappa$.

Remark 13: The formulated C-MRS MINLP problem is NP-hard in general (i.e. extremely computationally intensive), and thus may not be solved accurately in dense multi-source multi-relay networks $[36,37]$.

\section{Simulation Results}

This section presents simulation results of the proposed distributed multi-relay selection algorithms. In 
particular, the simulations assume $E_{B_{i}}=E_{\max }=150$ $\mathrm{mW}(\forall i \in\{1,2, \ldots, N\}), B_{\max }=1000 \mathrm{~mW}, N_{0}=$ $10^{-6} \mathrm{~W}, \nu=2.5, \rho=0.1$, and $\chi_{r_{k}}=0.001, \forall k \in$ $\{1,2, \ldots, K\}$. Moreover, the simulation results are averaged over 10,000 independent runs of randomly generated channel coefficients, which are assumed to be quasi-static during each communication frame but vary slowly from one frame to another, over a total of 50 frames. The simulated network topology assumes $N=$ 3 source and $K=4$ relay nodes, located as shown in Fig. 1. The rate and maximum value of energy arrivals under the Good and Bad relay states are given in Table 1. Furthermore, it is assumed that the initial beliefs of each relay is $p_{r_{k}}^{(0)}=0.5, \forall \theta_{r_{k}} \in\{G, B\}$ and $\forall k \in\{1,2,3,4\}$. It should be noted that the network topology is designed such that it introduces symmetry into the locations of the source and relay nodes, such that the results can be verified easily.

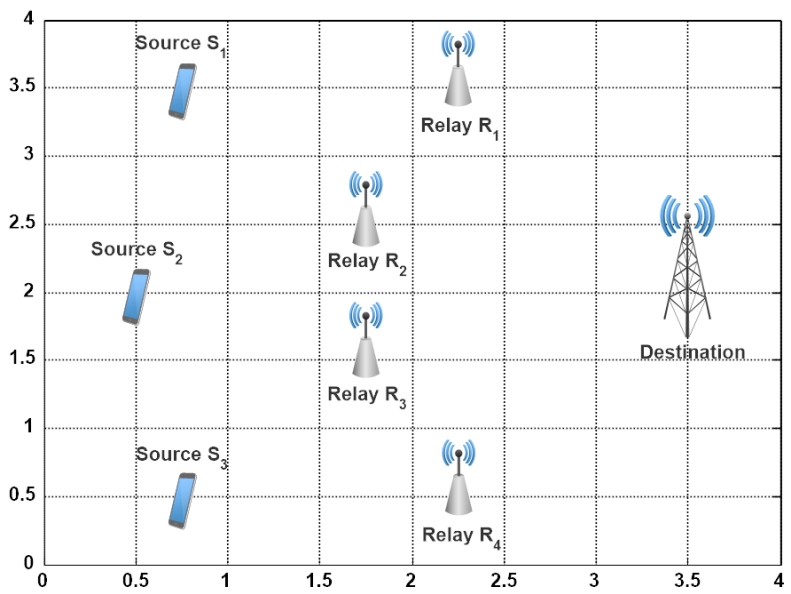

Fig. 1 Network Topology

\begin{tabular}{c|c||c|c|c|c}
\hline State & Values & $R_{1}$ & $R_{2}$ & $R_{3}$ & $R_{4}$ \\
\hline \multirow{2}{*}{ Good } & $\lambda_{r_{k}, G}$ (Arrivals/Time-Slot) & 5 & 6 & 6 & 5 \\
& $\mathcal{E}_{r_{k}, G}^{\max }(\mathrm{mW})$ & 3 & 4 & 4 & 3 \\
\hline \multirow{2}{*}{ Bad } & $\lambda_{r_{k}, B}$ (Arrivals/Time-Slot) & 2 & 3 & 3 & 2 \\
& $\mathcal{E}_{r_{k}, B}^{\max }(\mathrm{mW})$ & 1 & 2 & 2 & 1 \\
\hline
\end{tabular}

Table 1 Summary of Rate and Maximum Value of Energy Arrivals for Each Relay State

Remark 14: It is assumed that relays $R_{1}$ and $R_{4}$ are in bad state, while relays $R_{2}$ and $R_{3}$ are in good state.

Remark 15: To ensure fairness, the sequence of relay selections by source nodes in the BR-CS and BRUS algorithms has been performed in a round-robin fashion.

The proposed BR-CS and BR-US algorithms with NBL are compared with:
Centralized Multi-Relay Selection (C-MRS):

The C-MRS MINLP problem ${ }^{9}$ presented in Section 6 is compared for different maximum numbers of relay selections $\kappa$.

Learning-Based Multi-Relay Selection (L-MRS):

Each source node $S_{i}$ selects relays based on the updated beliefs about all relays $\mathbf{p}_{R}^{\zeta}$ in each communication frame $\zeta$; however, the selections of the previous source nodes are not considered. Thus, the expected utility of source node $S_{i}$ is calculated as [21]

$$
\begin{aligned}
& \mathbb{U}_{i, k}\left(\mathbf{p}_{r_{k}}^{\zeta}\right)= \\
& \quad \sum_{\theta_{r_{k}} \in \Theta} \sum_{\xi=0}^{\infty} \mathcal{U}_{i, k}\left(\xi, \mathcal{I}_{i, k}^{\zeta}\right) p_{\xi_{r_{k}}^{\zeta}}\left(\xi \mid \theta_{r_{k}}\right) p_{r_{k}}^{\zeta}\left(\theta_{r_{k}}\right) .
\end{aligned}
$$

\section{Myopic Multi-Relay Selection (M-MRS):}

In each communication frame $\zeta$, each source node $S_{i}$ selects relays based on the current observation $\mathbf{n}_{s_{i}}^{\zeta}$, without any learning (i.e. updated beliefs are not considered). Specifically, the expected utility of each source $S_{i}$ when selecting relay $R_{k}$ is determined as [21]

$$
\begin{aligned}
& \mathbb{U}_{i, k}\left(n_{s_{i}, r_{k}}^{\zeta}\right)= \\
& \quad \sum_{\theta_{r_{k}} \in \Theta} \sum_{\xi=0}^{\infty} \mathcal{U}_{i, k}\left(\xi, n_{s_{i}, r_{k}}^{\zeta}+\mathcal{I}_{i, k}^{\zeta}\right) p_{\xi_{r_{k}}^{\zeta}}\left(\xi \mid \theta_{r_{k}}\right) p_{r_{k}}^{0}\left(\theta_{r_{k}}\right),
\end{aligned}
$$

where $p_{r_{k}}^{0}\left(\theta_{r_{k}}\right)$ is the fixed initial belief about relay $R_{k}$ for state $\theta_{k}$.

\section{Random Multi-Relay Selection (R-MRS):}

Each source node randomly selects $\kappa$ relays in each communication frame without any bearing on the beliefs, energy arrivals, or other source nodes' selections.

In Fig. 2, the rate improvement of and sum-payment made by each source node under the different MRS algorithms are presented. Particularly, it can be seen that for the different values of $1 \leq \kappa \leq 3$, the C-MRS achieves the highest rate improvement for all source nodes. This is also met by the highest sum-payment made to the selected relays in return for their harvested energy that is utilized for cooperative transmission. This is attributed to the fact that the C-MRS selects the optimal set of relays to maximize its utility. It is also noticed that the BR-CS algorithm achieves the second best rate improvement for each source node,

9 The C-MRS optimization problem is solved using MIDACO [38], with tolerance set to 0.001 . 


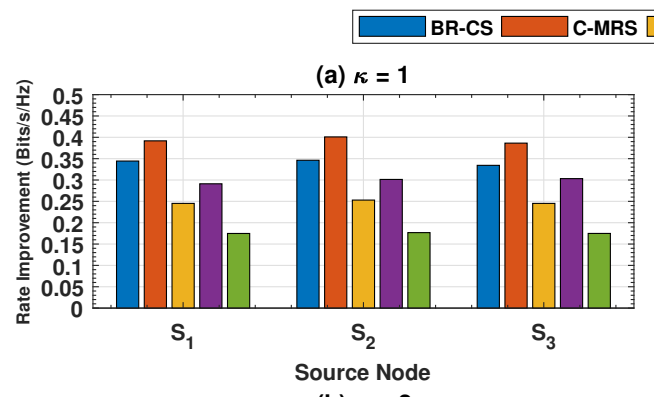

(b) $\kappa=2$

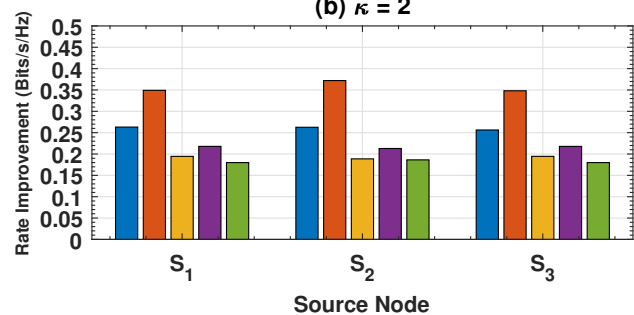

(c) $\kappa=3$

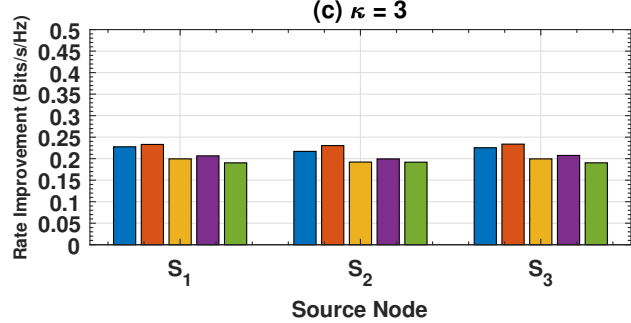

(d) $\kappa=4$
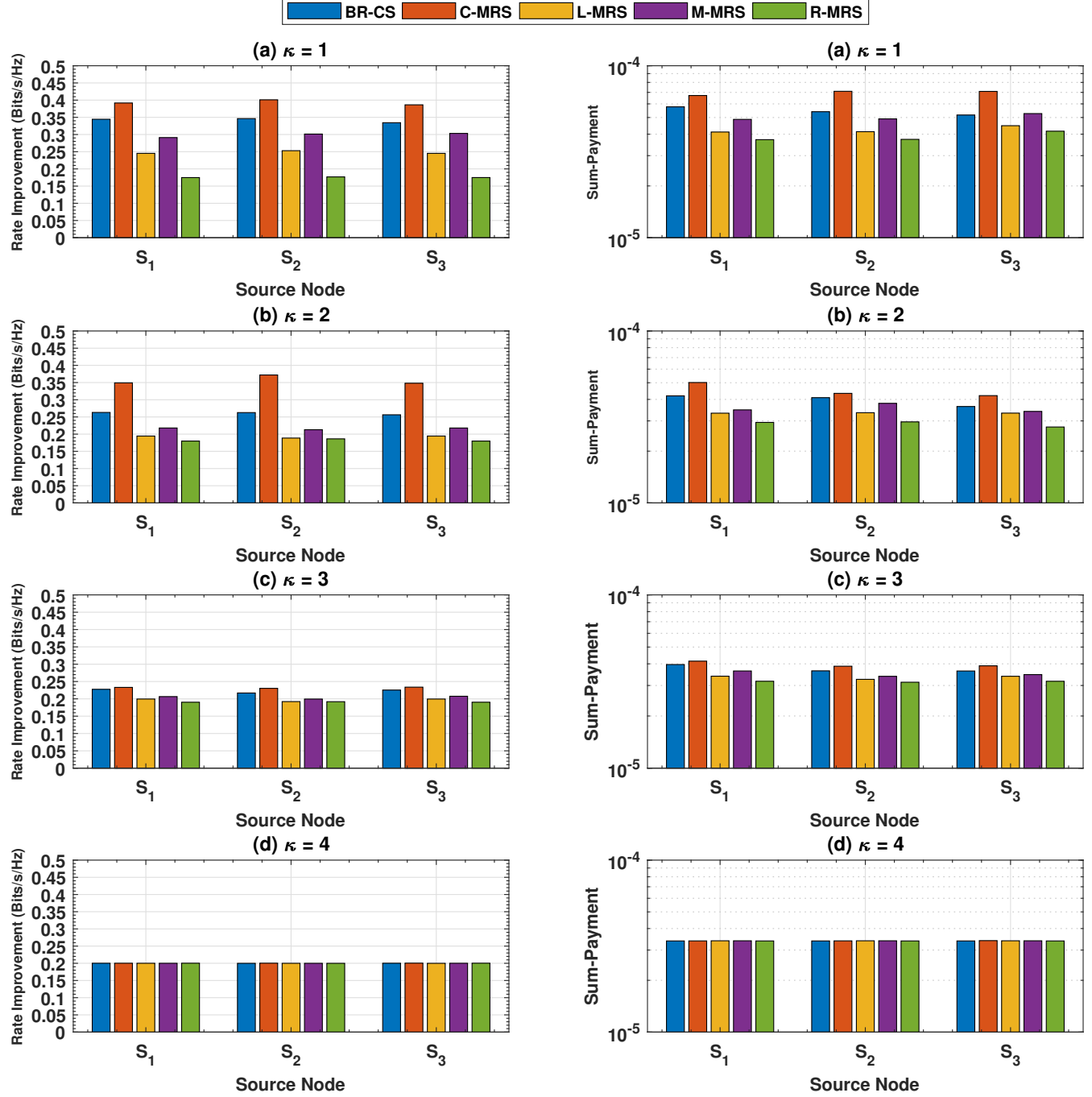

(b) $\kappa=2$

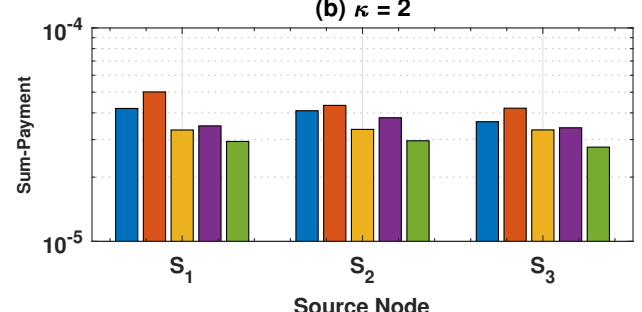

(c) $\kappa=3$

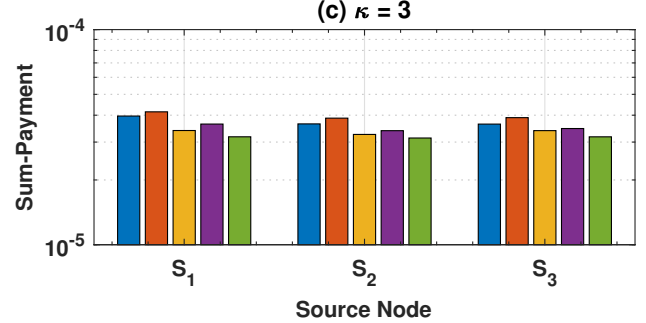

(d) $\kappa=4$

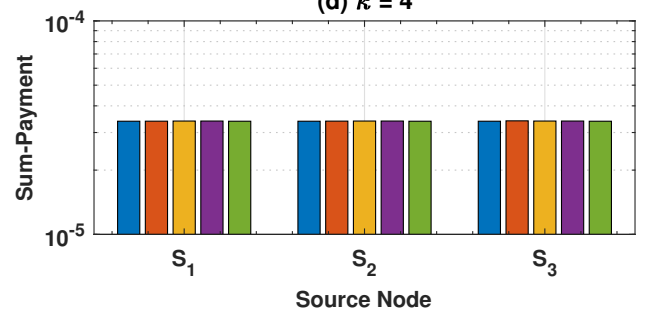

Fig. 2 Rate Improvement and Sum-Payment of Each Source Node for Different Values of $\kappa$ - BR-CS Algorithm

which is followed by the M-MRS, L-MRS, and RMRS algorithms, respectively. This is due to the fact that the proposed BR-CS algorithm incorporates both learning and current observations of relays selections of all source nodes, while the M-MRS is only based current observations (without learning), and the L-MRS is only based on updated beliefs via learning. Furthermore, the R-MRS yields the worst rate improvement per source node and also the lowest sum-payment since it randomly assigns relays to source nodes without taking into account the relay state (good or bad), or current number of source nodes selecting each relay. More importantly, the reason the C-MRS is superior to the BR-CS is that the latter algorithm takes a number of iterations to converge to the true beliefs (and probabilities) about the state of each relay, thus provides suboptimal relay selections in the first few iterations until convergence. For the case when $\kappa=4$ (i.e. all source nodes are allowed to select all $K=4$ relays), it can be seen that all the algorithms yield similar rate improvement and sum-payment results for each source node. Intuitively, this is due to the fact all source nodes can select all $k=4$ relays, hence the number of source nodes sharing each relay will be identical under the different algorithms. On the other hand, it is noticed that with the increase in the value of $\kappa$, the rate improvement of and sum-payment made by each source node decreases for the different algorithms. This is due to the fact that increasing $\kappa$ increases the average number of source nodes sharing each relay $n_{r_{k}}, \forall k \in\{1,2, \ldots, K\}$, which in turn decreases the amount of cooperative transmit energy per source node and thus leads to less rate improvement and less sum-payment (as per Remark 7). A similar observation is seen in Fig. 3 for the resulting utility per source node for the different values of $\kappa$ under the different MRS algorithms, which is due to the fact that the utility of each source node is equivalent to the rate improvement minus the sum-payment. 


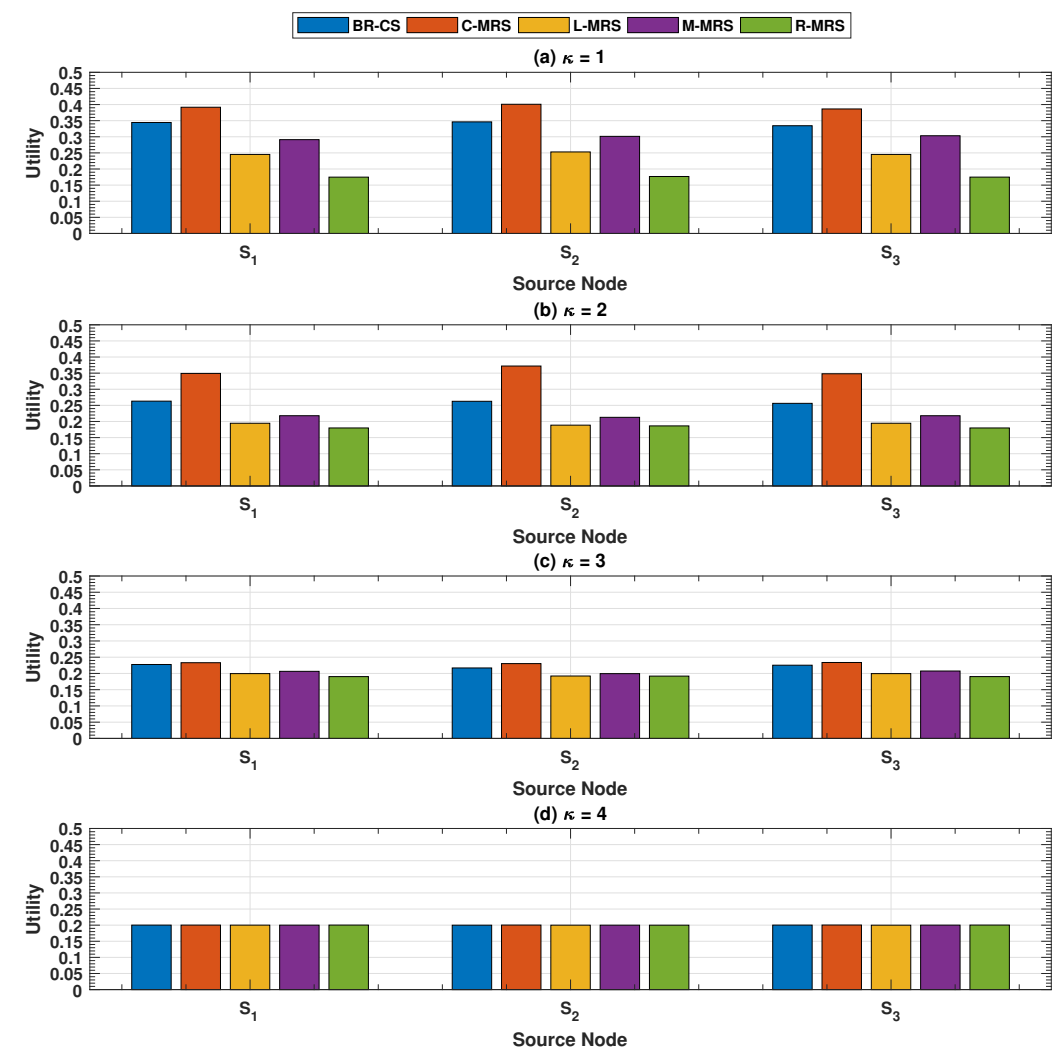

Fig. 3 Utility of Each Source Node for Different Values of $\kappa$ - BR-CS Algorithm
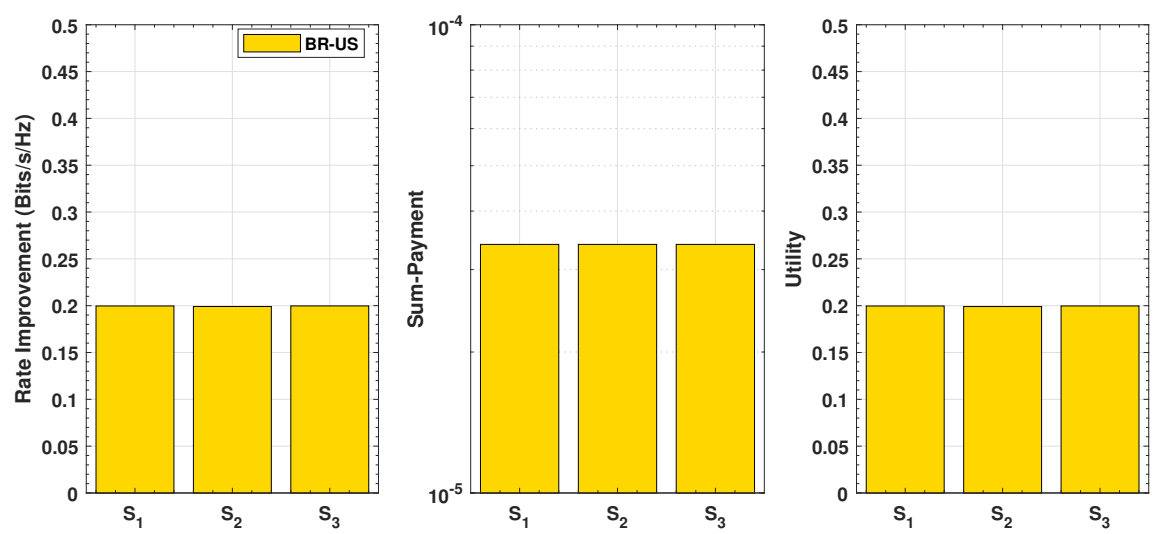

Fig. 4 Rate Improvement, Sum-Payment and Utility of Each Source Node - BR-US Algorithm

In Fig. 4, the rate-improvement of and sum-payment made (and the resulting utility) by each source node under the BR-US algorithm are demonstrated. It is clear to see that the rate improvement, sum-payment and utility results per source node are identical to those of the different MRS algorithms for $\kappa=4$ (see Figs. 2 and $3)$. This is also evident from the network sum-rate improvement under the different MRS algorithms shown in Fig. 5, which illustrates that the BR-CS algorithm yields higher network sum-rate improvement than the BR-US algorithm $\forall 1 \leq \kappa \leq 3$, but identical results for $\kappa=4$. Intuitively, this is because the BR-US allows the source nodes to select as many relays as possible (i.e. $\kappa=K=4$ ), and thus is equivalent to the BR-CS algorithm (as per Remark 10), and the other algorithms. This is also evident from Fig. 6, which demonstrates the average number of source nodes selecting each relay. Particularly, one can see that all $K=4$ relays are selected each by $N=3$ source nodes under the different MRS algorithms for $\kappa=4$.

Figs. $7 \mathrm{a}$ and $7 \mathrm{~b}$ illustrate the probability (and belief) of the good and bad state of each relay for $\kappa=1$, respectively, for the BR-CS algorithm. Specifically, in Fig. 7 a, it is clear that relays $R_{2}$ and $R_{3}$ converge to the 

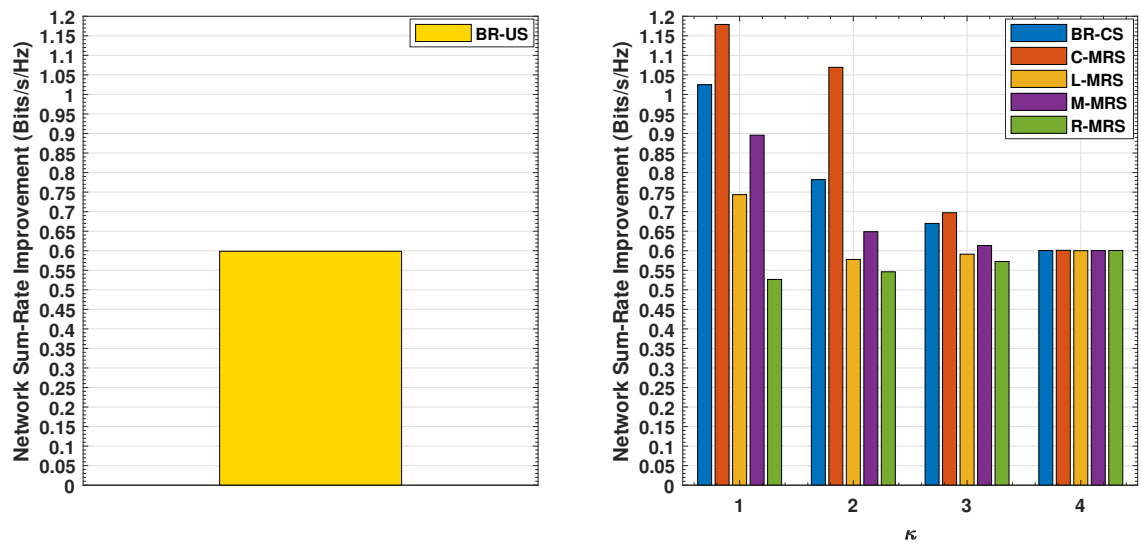

Fig. 5 Network Sum-Rate Improvement
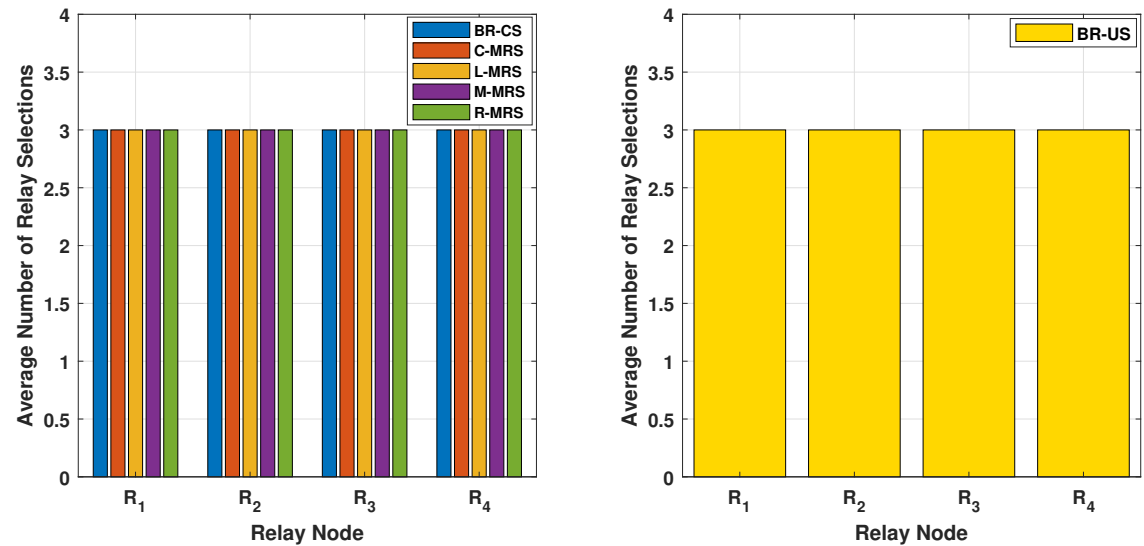

Fig. 6 Average Relay Selection of Each Relay for $\kappa=4$
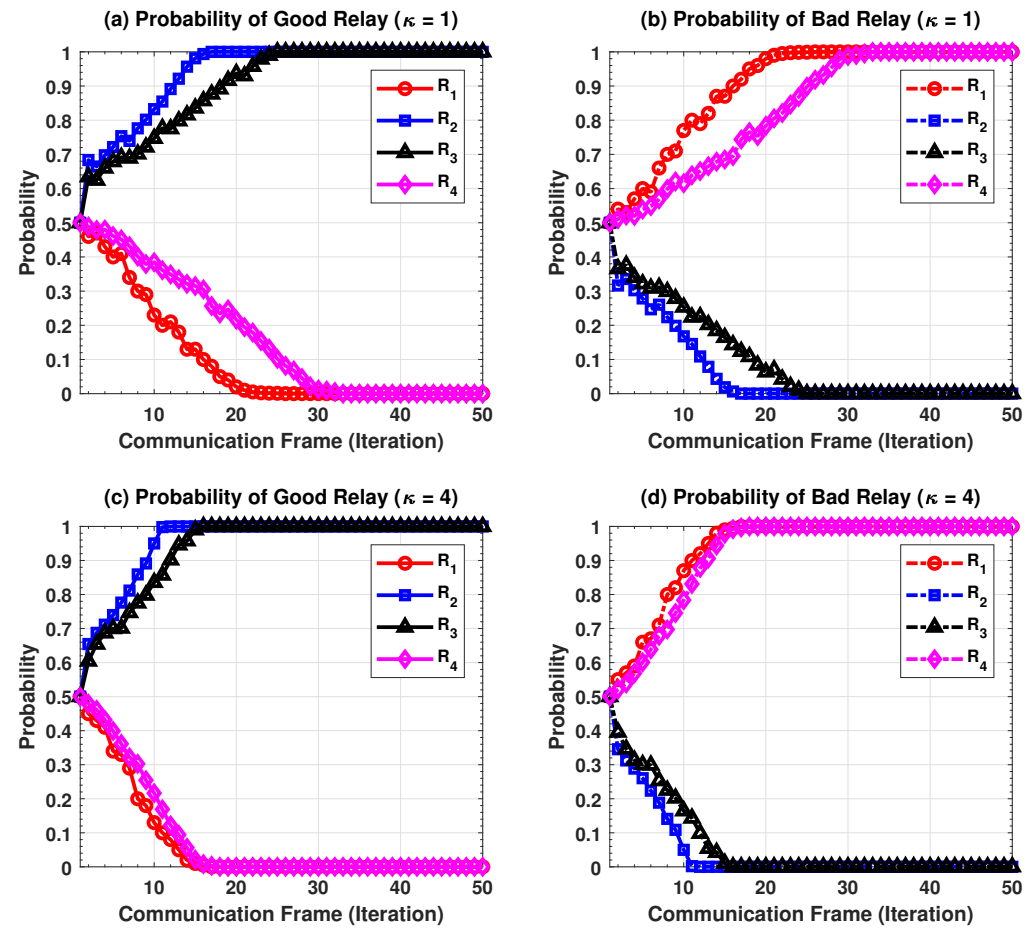

Fig. 7 Probability of Good and Bad Relay for Different Values of $\kappa$ - BR-CS Algorithm 

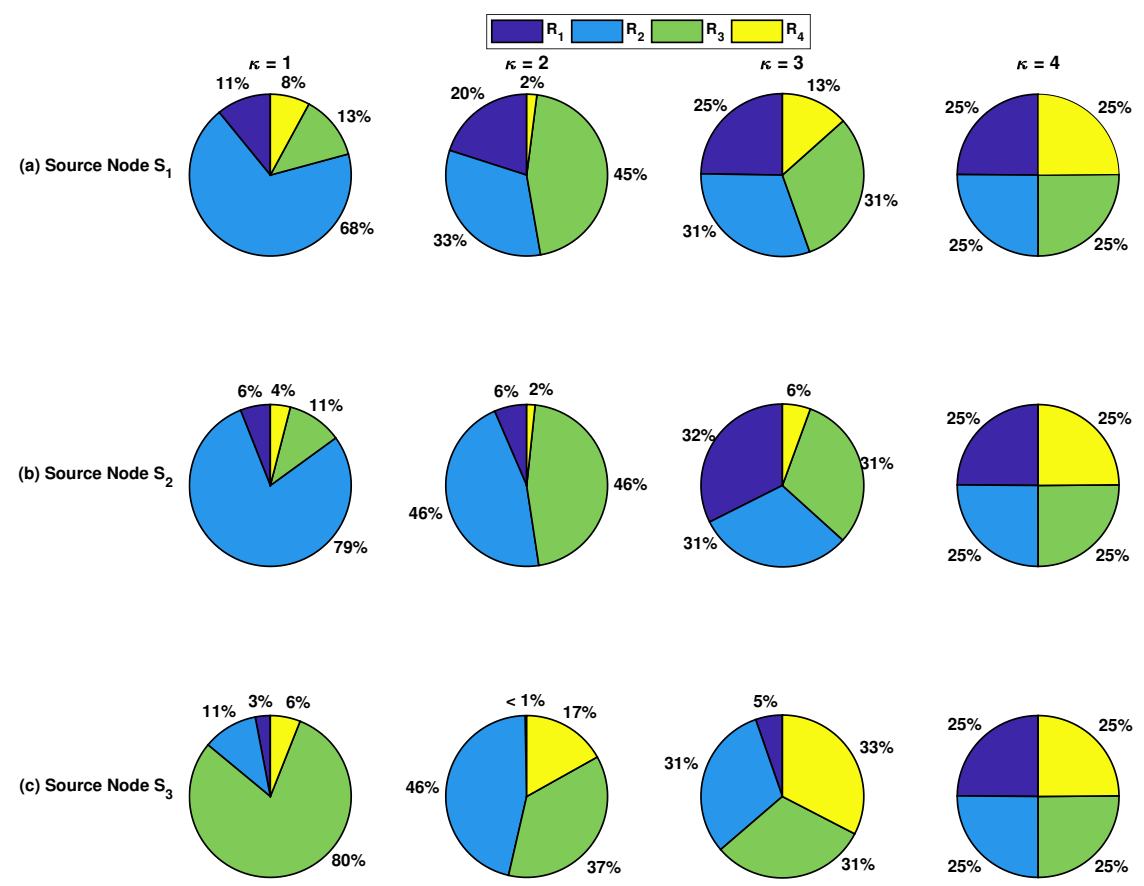

Fig. 8 Average Relay Selection of Each Relay Node by Each Source Node for Different Values of $\kappa$ - BR-CS Algorithm

probability of one for being good relays, while relays $R_{1}$ and $R_{4}$ converge to a probability of zero for being good relays (i.e. they are bad relays with probability of one). Contrarily, in Fig. 7b, relays $R_{2}$ and $R_{3}$ converge to a probability of zero for being bad relays, while relays $R_{1}$ and $R_{4}$ converge to a probability of one for being bad relays. This is in agreement with Remark 14. A similar observation is noticed in Figs. $7 \mathrm{c}$ and $7 \mathrm{~d}$, for $\kappa=4$. However, it can be seen that the convergence is faster when $\kappa=4$ than when $\kappa=1$. This is because the greater is the number of selected relays, the greater is the amount of inferred information, and hence the faster the belief is updated to reach the true state of each relay. A similar observation has been noticed for the BR-US algorithm to that of the BR-CS algorithm with $\kappa=4$, but is not shown to minimize redundancy.

In Fig. 8, the average relay selection by each source node under the BR-CS algorithm for different values of $\kappa$ is shown. One can see that for $\kappa=1$, source node $S_{1}$ selects relay $R_{2} 68 \%$ of the time and selects relay $R_{3}$ about $13 \%$ of the time. Similarly, source node $S_{2}$ selects relay $R_{2}$ about $79 \%$ of the time, and selects relay $R_{3}$ about $11 \%$ of the time. Lastly, source node $S_{3}$ selects relay $R_{3}$ about $80 \%$ of the time, and selects relay $R_{2}$ about $11 \%$ of the time. Clearly, all source nodes select relays $R_{2}$ and $R_{3}$ much more often than the other two relays. This is due to the fact that these two relays are in good state, and hence harvest more energy than the other two relays (as per Remark 14). A similar observation can be made for the case of $\kappa=2$. For $\kappa=3$, source node $S_{1}\left(S_{3}\right)$ selects relay $R_{4}\left(R_{1}\right)$ the least, since it is farthest from it. As for source node $S_{2}$, it selects relays $R_{1}, R_{2}$ and $R_{3}$ much more often than relay $R_{4}$, which is due to its order in selecting relays, as it is followed by source $S_{3}$ which selects relay $R_{4}$ more often than relay $R_{1}$. Lastly, for $\kappa=4$, it is evident that all relays are equally selected by all source nodes.

Fig. 9 illustrates the average worst-case complexity of the BR-CS and BR-US algorithms. It can be seen that the complexity of BR-CS algorithm increases drastically with the increase in $\kappa$. More importantly, the BR-CS algorithm is less complex than its BRUS algorithm only when $\kappa=1$. This in turn poses an important tradeoff between the BR-CS and BR-US in the sense that the BR-CS algorithm yields better network sum-rate improvement (and thus utility) than its counterpart BR-US algorithm with less complexity. However, for $1<\kappa \leq 4$, the BR-US algorithm is significantly less complex but yields lower network sum-rate improvement (and utility).

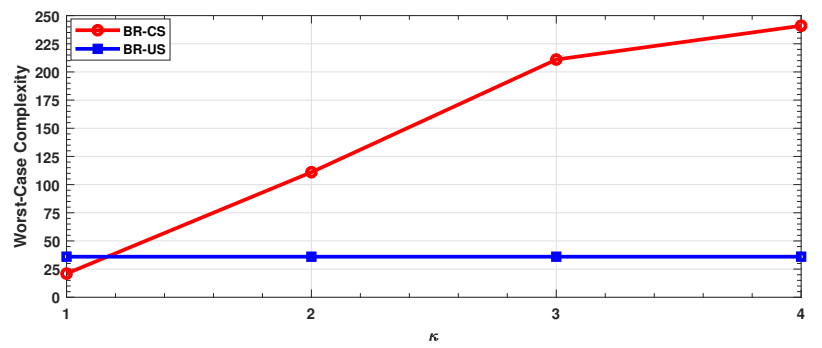

Fig. 9 Worst-Case Complexity for the BR-CS and BR-US Algorithms with $N=3$ and $K=4$ 
Finally, the superior performance of the BR-CS algorithm when $\kappa=1$ in comparison to $1<\kappa \leq 4$ is justified as follows. The multi-relay selection problem considered in this work is based on the assumption that each relay's transmit energy is equally distributed among the source nodes selecting it (as per Remark 6). Therefore, although setting $k=K=4$ achieves full diversity, it still yields sub-optimal performance [39], as the relay transmit energy is equally split among the source nodes (i.e. not optimally allocated). However, for the case when $\kappa=1$, the "best/optimal" relay is selected by each source node, which also exploits full diversity gains (as in the case of $\kappa=K$ ) [33]. However, in this case fewer source nodes are sharing each relay (on average), which implies a greater share of relay transmit energy per source node, and hence the greater the resulting rate improvement (and utility). This is in agreement with observations made in [39] and [33], which attest that optimal "single" relay selection outperforms multi-relay selection (even with optimal power allocation). Last-but-not-least, reducing the number of transmitting relays via optimal "single" relay selection can help mitigate the adverse effects of channel estimation errors and timing offsets [33]. Hence, the number of relay selections must be constrained to the minimum, so as to reduce complexity and fully exploit diversity gains.

\section{Conclusions}

In this paper, the problem of distributed multi-relay selection with negative network externality in energyharvesting cooperative wireless networks has been studied from a game-theoretic perspective. Specifically, the IBG model has been utilized to devise two best-response recursive distributed algorithms for the scenarios of constrained and unconstrained relay selections, with nonBayesian learning so as to determine the unknown relays' energy states. The proposed distributed algorithms have been shown to allow source nodes to make multirelay selection decisions while guaranteeing subgame perfect Nash equilibrium. Moreover the proposed algorithms have been compared with centralized, learningbased, myopic and random multi-relay selection schemes, where it has been shown that the distributed BR-CS algorithm yields comparable performance (i.e. network sum-rate improvement, sum-payment and utility) to the centralized multi-relay selection. More importantly, the BR-CS algorithm is shown to be superior to the BR-US in terms of network sum-rate improvement (and utility) when it is constrained to relay selections that are less than the maximum number of available relays, but at the expense of higher complexity. Consequently, the number of relay selections of the BR-CS algorithm must be constrained to the minimum, so as to reduce complexity and fully exploit diversity gains.

It should be noted that the work presented in this paper can be extended in several directions. For instance, equal power allocation has been assumed at each relay; however, it would be interesting to consider the problem of distributed relay selection with optimal power allocation. Moreover, in this work, TDMA has been adopted for data transmission, where each source node has been allocated a dedicated time-slot (i.e. an orthogonal transmission scheme). Thus, it would be of great importance to consider a more sophisticated nonorthogonal multiple-access scheme, so as to improve spectrum utilization, while taking into account interuser interference. Furthermore, the communication signalling and overheads across the source and relay nodes are yet to fully studied. Specifically, for the devised algorithms to work in practice, modifications to the physical, data link, and network layers must be incorporated. This entails multi-layer simulation and experimentation to acquire realistic assessment of the proposed algorithms, while accounting for potential packet losses and retransmissions.

\section{References}

1. Lu X., Wang P., Niyato D., and Han Z.: 'Wireless networks with RF energy harvesting: A contemporary survey', IEEE Communications Surveys and Tutorials, 17 (2), pp. 757-789, 2015.

2. Ulukus S., Yener A., Erkip E., Simeone O., Zorzi M., Grover P., and Huang K.: 'Energy harvesting wireless communications: A review of recent advances', IEEE Journal on Selected Areas in Communications, 33 (3), pp. 360-381, 2015.

3. Liu K. J. R., Sadek A. K., Su W., and Kwasinski A.: 'Cooperation communications and networking' (Cambridge University Press, 2008).

4. Baidas M. W.: 'Cooperation in wireless networks: a gametheoretic framework with reinforcement learning', IET Communications, 8 (5), pp. 740-753, 2014.

5. Bahbahani M. S., Baidas M. W., and Alsusa E.: 'A distributed political coalition formation framework for multi-relay selection in cooperative wireless networks', IEEE Transactions on Wireless Communications, 14 (12), pp. 6869-6882, 2015.

6. Bahbahani M. S., and Alsusa E.: 'Joint cost-sharing and multi-relay selection for two-way networks using a pricing game', Proc. of IEEE Wireless Communications and Networking Conference (WCNC), pp. 1-6, 2016.

7. Bahbahani M. S., and Alsusa E.: 'Relay selection for energy harvesting relay networks using a repeated game', Proc. of IEEE Wireless Communications and Networking Conference (WCNC), pp. 1-6, 2016.

8. Baidas M. W., and Bahbahani M. S.: 'Game-theoretic modeling and analysis of relay selection in cooperative wireless networks', Wireless Communications and Mobile Computing, 16 (5), pp. 500-518, 2016. 
9. Ding L., Shen L., Liu D., Xu K., and Xu Y.: 'A game theoretic learning solution for distributed relay selection on throughput maximization', Wireless Networks, 23 (6), pp. $1757-1766,2017$.

10. Baidas M. W., and Alsusa E.: 'Power allocation, relay selection, and energy cooperation strategies in energy harvesting cooperative wireless networks', Wireless Communications and Mobile Computing, 16 (4), pp. 2065-2082, 2016.

11. Yang D., Zhu C., Xiao L., Shen X., and Zhang T.: 'An energy-efficient scheme for multirelay cooperative networks with energy harvesting', Mobile Information Systems, article ID: 5618935, 2016, DOI: 10.1155/2016/5618935.

12. Do N. T., Bao V. N. Q., and An B.: 'Outage performance analysis of relay selection schemes in wireless energy harvesting cooperative networks over non-identical Rayleigh fading channels', Sensors, 16 (3), 295, 2016.

13. Do N. T., Bao V. N. Q., and An B.: 'A relay selection protocol for wireless energy harvesting relay networks', Proc. of IEEE International Conference on Advanced Technologies for Communications (ATC), pp. 243-247, 2015.

14. Son P. N., and Kong H. Y.: 'Energy-harvesting relay selection schemes for decode-and-forward dual-hop networks', IEICE Transactions on Communications, 98 (12), pp. 2485-2495, 2015.

15. Gu Y., Chen H., Li Y., Liang Y., and Vucetic B.: 'Distributed multi-relay selection in accumulate-then-forward energy harvesting relay networks', IEEE Transactions on Green Communications and Networking, 2 (1), pp. 7486, 2017.

16. Zheng L., Zhai C., and Liu J.: 'Alternate energy harvesting and information relaying in cooperative AF networks', Telecommunication Systems, 68 (3), pp. 523-533, 2018.

17. Song X., Xu S., Xie Z., and Han X.: 'Joint optimal power allocation and relay selection scheme in energyharvesting two-way relaying networks', Future Internet, 11, 47, 2019, DOI: 10.3390/fi11020047.

18. Nguyen T. N., Minh T. H. Q., Tran P. T., Voznak M., Duy T. T., Nguyen T.-L., and Tin P. T.: 'Performance enhancement for energy harvesting based two-way relay protocols in wireless ad-hoc networks with partial and full relay selection methods', Ad Hoc Networks, 84 (1), pp. 178-187, 2019.

19. Bao V. N. Q., and Van N. T.: 'Incremental relaying networks with energy harvesting relay selection: Performance analysis', Emerging Telecommunications Technologies, 29 (12), e3483, 2018.

20. Andrawes A., Nordin R., and Ismail M.: 'Wireless energy harvesting with cooperative relaying under the best relay selection scheme', Energies, 12 (5), 892, 2019, DOI: $10.3390 /$ en12050892.

21. Jiang C., Chen Y., Gao Y., and Liu K. J. R.: 'Indian buffet game with negative network externality and nonbayesian social learning', IEEE Transactions on Systems, Man, and Cybernetics: Systems, 45 (4), pp. 609-623, 2015 .

22. Griffiths T. L., and Ghahramani Z.: 'The Indian buffet process: An introduction and review', Journal of $\mathrm{Ma}$ chine Learning, 12, pp. 1185-1224, 2011.

23. Zhang Y., Pan E., Song L., Saad W., Dawy Z., and Han Z., "Social Network Aware Device-to-Device Communication in Wireless Networks", IEEE Transactions on Wireless Communications, 14 (1), pp. 177-190, 2015.
24. Hung H. J., Ho T. Y., Lee S. Y., Yang C. Y., and Yang D. N.: 'Relay selection for heterogeneous cellular networks with renewable green energy sources', IEEE Transactions on Mobile Computing, 17 (3), pp. 661-674, 2018.

25. Lee S. Y., Liu C. Y., Chang M. K., Yang D. N., and Hong Y. W. P.: 'Cooperative multicasting in renewable energy enhanced relay networks - Expending more power to save energy', IEEE Transactions on Wireless Communications, 15 (1), pp. 753-768, 2016.

26. Zhe W., Wang X., Aldiab M., and Jaber T.: 'User association for energy harvesting relay stations in cellular networks', EURASIP Journal on Wirless Communications and Networking, 264, pp. 1-12, 2015.

27. Miridakis N. I., Tsiftsis T. A., Alexandropolous G. C., and Debbah M.: 'Green cognitive relaying: Opportunistically switching between data transmission and energy harvesting', IEEE Journal on Selected Areas in Communications, 34 (12), pp. 3725-3738, 2016.

28. Renyi A.: 'Probability Theory' (North-Holland, Amsterdam, 1970).

29. Buonocore A., Pirozzi E., and Caputo L.: 'A note on the sum of uniform random variables', Elsevier Statistics and Probability Letters, 79 (10), pp. 2092-2097, 2009.

30. Ross S. M.: 'Introduction to Probability Models, 10th Ed.' (Academic Press, 2009).

31. Tsao C. K.: 'Distribution of the sum in random samples from a discrete population', Annals of Mathematical Statistics, 27 (3), pp. 703-712, 1956.

32. Adelson R. M.: 'Compound poisson distributions', Operations Research, 17 (1), pp. 73-75, 1966.

33. Baidas M. W., and MacKenzie A. B.: 'Many-to-many space-time network coding for amplify-and-forward cooperation networks: Node selection and performance analysis', EURASIP Journal on Wireless Communications and Networking, 48, pp. 1-7, 2014, DOI: 10.1186/16871499-2014-48.

34. Baidas M. W., and MacKenzie A. B.: 'Auction-based power allocation for many-to-one cooperative wireless networks', Proc. of IEEE International Wireless Communications and Mobile Computing Conference, pp. 1677-1682, 2011.

35. Osborne J.: 'An introduction to game theory' (Oxford University Press, 2003).

36. Bonami P., Kilinc M., and Linderoth J.: 'Algorithms and software for convex mixed integer nonlinear programs', The IMA Volumes in Mathematics and its Applications, 154, pp. 1-39, 2012.

37. Nowak L.: 'Relaxation and decomposition methods for mixed integer nonlinear programming' (Spring Science and Business Media - Birkhauser Verlag, Basel, 2005).

38. Schlueter M.: 'MIDACO Software Performance on Interplanetary Trajectory Benchmarks', Advances in Space Research, 54 (4), pp. 744-754, 2014.

39. Zhao Y., Adve R., and Lim T.: 'Improving amplify-andforward relay networks: optimal power allocation versus selection', IEEE Transactions on Wireless Communications, 6 (8), pp. 3114-3123, 2007. 